\title{
Numerical Assessment of an Anisotropic Porous Metal Plasticity Model
}

\author{
S. M. Keralavarma ${ }^{1}$ and A. A. Benzerga ${ }^{2,3}$ \\ ${ }^{1}$ Department of Aerospace Engineering, Indian Institute of Technology Madras \\ Chennai 600036, India \\ ${ }^{2}$ Department of Aerospace Engineering, Texas A\&M University \\ College Station, TX 77843-3141, USA \\ ${ }^{3}$ Department of Materials Science $\mathscr{6}$ Engineering, Texas A $6 M$ University \\ College Station, TX 77843-3141, USA
}

\begin{abstract}
The objective of this paper is to perform numerical assessment of a micromechanical model of porous metal plasticity developed previously by the authors. First, upper bound estimates for the yield loci are computed using homogenization and limit analysis of a spheroidal representative volume element containing a confocal spheroidal void, neglecting elasticity. Unlike in the development of the analytical model, the computational limit analysis is performed without recourse to approximations so that the obtained yield loci are rigorous upper bounds for the true criterion. Next, the model's macroscopic dilatancy at incipient plastic flow is compared against that of the numerical limit analysis approach. Finally, finite-element calculations, with elasticity included, are presented for transversely isotropic porous unit-cells loaded axisymmetrically. The effective stress-strain response as well as evolution of the unit-cell porosity and void aspect ratio are compared with theoretical predictions.
\end{abstract}

Keywords: Ductile Fracture; Elastic-Plastic Porous Materials; Void Growth; Homogenization; Limit Analysis; Upper Bound Theory 


\section{Introduction}

The primary mechanism of ductile fracture is the growth and coalescence of microvoids or blunted micro-cracks. In metals, these nucleate from hard inclusions and second phase particles [1], or sometimes at slip- or twin-boundaries and intersections [26]. Microvoid growth and coalescence are natural outcomes in porous metal plasticity [4]; also see Benzerga and Leblond [8] for an extensive review. Earlier models were isotropic and only accounted for void growth $[18,41]$ with subsequent extensions to coalescence being heuristic [47]. Later, porous metal plasticity models accounting for anisotropy and coalescence have been developed with increasing degree of accuracy, e.g., [13, 5, 31, 32, 9].

Of particular interest are models having a micromechanically-derived capability to couple void shape and plastic anisotropy [23, 34, 24]. Modeling void shape evolution is essential to various fundamental problems in ductile fracture mechanics such as: (i) developing criteria for the onset of void coalescence $[6,38,3]$; (ii) accounting for damage and fracture anisotropy [7]; and (iii) understanding damage accumulation under complex loading conditions, for example void rotation in a shear field [36, 46] (obviously, rotation of a void is meaningful if the void has a non-spherical shape). On the other hand, modeling plastic anisotropy is essential to a host of engineering materials, notably aluminum alloys and hexagonal close packed materials such as magnesium, titanium and zirconium alloys. What is of particular importance is that the net rate of void growth in an anisotropic material can be virtually suppressed or enhanced, under any stress state, depending on the degree of anisotropy [5, 25]. This fact begins to be recognized in the mechanics literature but remains to be taken fully advantage of in designing damage-tolerant, fracture-resistant materials.

The constitutive models of anisotropic porous metal plasticity were developed based on nonlinear homogenization combined with the theory of limit analysis. Their derivation typically involves consideration of a hollow spheroidal representative volume element (RVE) made of a Hill orthotropic material and various kinematically admissible trial velocity fields at the microscale [8]. For instance, Monchiet et al. [34] developed a model based on consideration of the velocity fields used by Gologanu et al. $[13,14]$ in their earlier versions of the GLD model, and Keralavarma and Benzerga [23] developed an improved solution using a broader space of velocity fields [29] also used by Gologanu et al. [15] in their improved GLD model. The model is, however, restricted to axisymmetric loadings and microstructures for which the void axis is aligned with one direction of material orthotropy. Later, Keralavarma and Benzerga [24] developed a generalized model applicable to arbitrary loadings and void orientations. This model thus constitutes a generalization of the GLD model to plastically anisotropic matrices and also a generalization of Benzerga and Besson's (2001) model to spheriodal voids. Evolution equations were supplied for the void volume fraction, void aspect ratio and void rotation. It is worth noting that plastic potentials for ellipsoidal voids in an isotropic matrix have been previously derived using an alternate non-linear homogenization procedure by Ponte Castañeda and Zaidman [40] and later improved by Danas and Ponte Castañeda [11]. However, neither of these works considered the case of anisotropic matrices. Within a similar variational framework Han et al. [19] and Paux et al. [39] have recently proposed yield criteria for porous single crys- 
tals. These models inherently account for plastic anisotropy effects at the crystal level but only for spherical voids. Other works have addressed the problem computationally. For example, Yerra et al. [48] investigated the effects of plastic flow anisotropy using a crystal plasticity description of the matrix to study the growth of spherical voids in single crystals. Interestingly, they noted that when a Hill criterion is fit to the crystal plasticity model their results can be rationalized for the most part on the basis of the BenzergaBesson model (2001). More recently, Lebensohn et al. [28] studied the growth of initially spherical voids in a polycrystalline matrix by means of a fast Fourier transform formulation. When fully developed, such results will provide a basis for assessing anisotropic porous metal plasticity models. Also, crack-void interactions were investigated in textured polycrystals [43]. However, for these more refined descriptions of matrix plasticity, closed form yield criteria have not been developed, presumably due to the analytical complexity of the homogenization problem.

The objective of the present paper is to perform a detailed numerical assessment of the approximate analytical model of Keralavarma and Benzerga [24]. The model considers aligned spheroidal voids in a Hill orthotropic matrix. The assumption of a spheroidal void shape entails some restrictions, although the problem is sufficiently general for the purpose of illustrating coupled effects of void shape and matrix anisotropy on ductile damage evolution. A brief summary of the analytical model is presented in section 2 for ease of reference. The performance of the model is assessed using two different approaches. In section 3, a numerical method is developed to compute upper-bound yield loci for anisotropic materials subjected to axisymmetric stress states following a limit analysis procedure using a large number of trial velocity fields derived from the incompressible axisymmetric velocity fields proposed by Lee and Mear [29]. Due to limitations of the trial velocity fields employed, tight upper bound loci are only guaranteed in the case of fully axisymmetric problems, even though rigorous bounds are obtained in all cases. Recently, a finite-element based limit analysis method has been proposed that obviates the need to choose trial velocity fields a priori [35], albeit at a higher computational cost. In section 4 , the analytical yield criterion is validated by comparison with these numerically derived upper bound yield loci. Additional results for the macroscopic dilatancy due to void growth at incipient yielding, obtained from the normality property of plastic flow, are also compared. In section 5, the analytical model is integrated for specified loading paths and the evolution equations for the microstructural variables are validated by comparing the model predictions with finite-element predictions for the same using micromechanical unit-cells.

\section{Model Synopsis}

In Keralavarma and Benzerga [24], the framework of Hill-Mandel homogenization $[21,33]$ and limit-analysis was used to derive an approximate analytical yield criterion for anisotropic porous materials, containing spheroidal voids embedded in a Hill-type orthotropic matrix [20]. The kinematic approach of homogenization was used, following previous works on void shape effects [15] and material anisotropy effects [5], wherein the representative volume element is subjected to homogeneous deformation rate boundary 


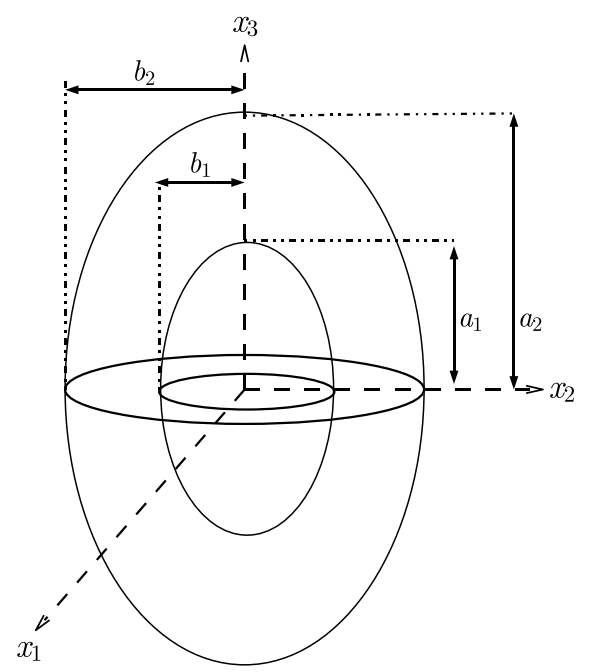

(a)

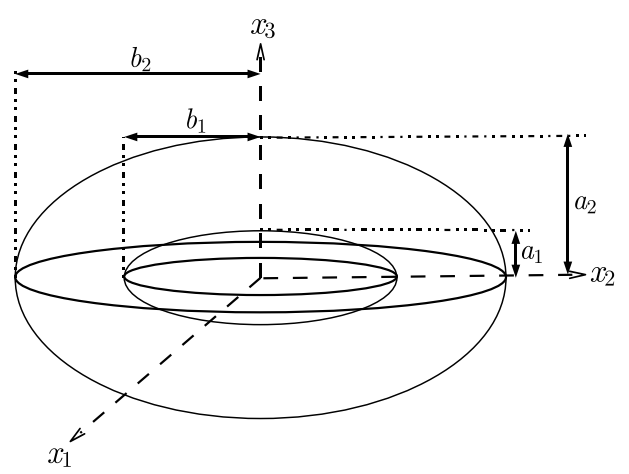

(b)

Figure 1: Spheroidal representative volume elements containing confocal spheroidal voids: (a) prolate (b) oblate.

conditions. The RVE was chosen to consist of a thick spheroidal shell containing a confocal spheroidal void, as illustrated in Fig. 1. Evolution laws were also derived for the microstructural variables, porosity, void aspect ratio and orientation. The main results are summarized here for completeness.

\subsection{Yield Criterion}

Following the Hill-Mandel homogenization approach, the macroscopic or 'average' stress, $\boldsymbol{\Sigma}$, and deformation rate, $\mathbf{D}$, for the RVE are given by

$$
\Sigma=\langle\boldsymbol{\sigma}\rangle_{\Omega}, \quad \mathbf{D}=\langle\mathbf{d}\rangle_{\Omega}
$$

where $\boldsymbol{\sigma}$ and $\mathbf{d}$ are the corresponding microscopic fields, $\Omega$ represents the volume of the RVE and the notation $\langle\cdot\rangle_{\Omega}$ represents the average over the volume of the RVE. An approximate limit-analysis assuming a rigid ideal plastic behavior for the matrix and using a limited set of trial velocity fields yields the following closed form expression for the macroscopic yield surface in stress space,

$$
\mathcal{F}(\boldsymbol{\Sigma})=C \frac{3}{2} \frac{\boldsymbol{\Sigma}: \mathbb{H}: \boldsymbol{\Sigma}}{\sigma_{0}^{2}}+2(g+1)(g+f) \cosh \left(\kappa \frac{\boldsymbol{\Sigma}: \mathbf{X}}{\sigma_{0}}\right)-(g+1)^{2}-(g+f)^{2}=0
$$

where $\sigma_{0}$ denotes the yield stress of the matrix in a reference direction and the fourth order tensor, $\mathbb{H}$, denotes the macroscopic plastic anisotropy tensor, defined by

$$
\mathbb{H} \equiv \mathbb{p}+\eta(\mathbf{X} \otimes \mathbf{Q}+\mathbf{Q} \otimes \mathbf{X})
$$

The plastic anisotropy of the matrix enters the criterion above via the Hill tensor, $\mathbb{p}$, and a tensor $\hat{\mathbb{p}}$ which is a pseudo-inverse of $\mathbb{p}$ via the relation $\mathbb{p}: \hat{\mathbb{p}}=\hat{\mathbb{p}}: \mathbb{p}=\mathbb{J}$. Here, $\mathbb{J}$ is 
the deviatoric projection operator given by $\mathbb{J}=\mathbb{1}-\frac{1}{3} \mathbf{1} \otimes \mathbf{1}$, where $\mathbb{1}$ and $\mathbf{1}$ are the fourth and second order identity tensors respectively. The tensors $\mathbb{p}$ and $\hat{\mathbb{P}}$ may be related to corresponding anisotropy tensors in deviatoric stress space $\mathbb{h}$ and $\hat{\mathbb{h}}$ by $\mathbb{J}: \mathbb{h}: \mathbb{J}=\mathbb{p}$ and $\mathbb{J}: \hat{\mathbb{h}}: \mathbb{J}=\hat{\mathbb{P}}[$ see 5$]$. The tensors $\mathbf{X}$ and $\mathbf{Q}$ are tied to the void orientation by

$$
\begin{gathered}
\mathbf{X} \equiv \alpha_{2}\left(\underline{e}_{1} \otimes \underline{e}_{1}+\underline{e}_{2} \otimes \underline{e}_{2}\right)+\left(1-2 \alpha_{2}\right) \underline{e}_{3} \otimes \underline{e}_{3} \\
\mathbf{Q} \equiv-\frac{1}{2}\left(\underline{e}_{1} \otimes \underline{e}_{1}+\underline{e}_{2} \otimes \underline{e}_{2}\right)+\underline{e}_{3} \otimes \underline{e}_{3}
\end{gathered}
$$

where $\left(\underline{e}_{1}, \underline{e}_{2}, \underline{e}_{3}\right)$ is a Cartesian frame introduced in Fig. 1 with $\underline{e}_{3}$ aligned with the void axis and the directions of $\underline{e}_{1}, \underline{e}_{2}$ chosen arbitrarily.

In addition to the anisotropy tensor, $\mathfrak{h}$, and the void orientation, the criterion depends on two non-dimensional variables that characterize the microstructure, the porosity, $f=$ $\frac{a_{1} b_{1}^{2}}{a_{2} b_{2}^{2}}$, and the void aspect ratio, $w=a_{1} / b_{1}$. The variables $f$ and $w$ are related to the eccentricities of the void, $e_{1}$, and the RVE, $e_{2}$, by

$$
\begin{gathered}
\frac{1}{1-e_{1}^{2}}= \begin{cases}w^{2} & (\mathrm{p}) \\
1 / w^{2} & (\mathrm{o})\end{cases} \\
\frac{\left(1-e_{2}^{2}\right)^{n}}{e_{2}^{3}}=\frac{1}{f} \frac{\left(1-e_{1}^{2}\right)^{n}}{e_{1}^{3}}, \quad n= \begin{cases}1 & (\mathrm{p}) \\
1 / 2 & (\mathrm{o})\end{cases}
\end{gathered}
$$

where the abbreviations (p) and (o) stand for prolate and oblate respectively. The parameter $g$ that appears in (2) may be interpreted as a 'porosity-like' quantity that takes non-zero values only for oblate void shapes, given by

$$
g \equiv\left\{\begin{array}{cc}
0 & (\mathrm{p}) \\
\frac{e_{2}^{3}}{\sqrt{1-e_{2}^{2}}} & (\mathrm{o})
\end{array}\right.
$$

In particular, a non-zero value of $g$ for penny-shaped cracks, which is a limiting configuration of an oblate void as the porosity tends to zero, allows for predictions of porosity growth corresponding to the opening of the crack under certain types of loading.

The parameters $C, \eta, \kappa$ and $\alpha_{2}$ that appear in the yield criterion (2) are functions of the microstructural variables, $f$ and $w$, defined above. In addition, these depend on material anisotropy via three scalar anisotropy factors given by

$$
\begin{gathered}
h=2\left[\frac{2\left(\hat{h}_{11}+\hat{h}_{22}+\hat{h}_{33}-\hat{h}_{23}-\hat{h}_{31}-\hat{h}_{12}\right)+3\left(\hat{h}_{44}+\hat{h}_{55}+\hat{h}_{66}\right)}{15}\right]^{1 / 2} \\
h_{q}=\frac{\hat{h}_{11}+\hat{h}_{22}+4 \hat{h}_{33}-4 \hat{h}_{23}-4 \hat{h}_{31}+2 \hat{h}_{12}}{6}, \quad h_{t} \equiv h^{2}-\frac{3}{4}\left(\hat{h}_{11}+\hat{h}_{22}+2 \hat{h}_{66}-2 \hat{h}_{12}\right)
\end{gathered}
$$

where $\hat{h}_{i j}$ denote the components of the fourth order tensor $\hat{\mathrm{h}}$, expressed in Voigt notation, in the frame $\left(\underline{e}_{1}, \underline{e}_{2}, \underline{e}_{3}\right)$ of Fig. 1. It may be demonstrated that the parameter $h$ is 
an invariant of the fourth order tensor $\hat{\mathrm{h}}$ while the values of $h_{q}$ and $h_{t}$ are invariant with respect to arbitrary coordinate rotations about the void axis $\underline{e}_{3}$, as required. The expressions for the model parameters as functions of $f, w$ and the above defined anisotropy factors are provided in Appendix A.

\subsection{Microstructure Evolution Laws}

Numerical computations using the model requires the introduction of elasticity, which is accounted for using an additive decomposition of the deformation rate tensor into elastic and plastic parts, $\mathbf{D}=\mathbf{D}^{e}+\mathbf{D}^{p}$. Assuming small elastic strains, a hypoelastic constitutive law may be used to write $\mathbf{D}^{e}=\mathbb{C}^{-1}: \dot{\boldsymbol{\Sigma}}$, where $\dot{\boldsymbol{\Sigma}}$ denotes some objective measure of the stress rate and $\mathbb{C}$ the tensor of elasticity. The macroscopic plastic strain rate may be derived from the yield potential using the normality flow rule $[21,18]$, i.e.

$$
\mathbf{D}^{p}=\Lambda \frac{\partial \mathcal{F}}{\partial \boldsymbol{\Sigma}}(\boldsymbol{\Sigma})
$$

where $\mathcal{F}$ denotes the yield function of equation (2) and $\Lambda$ denotes the plastic multiplier. The evolution law for the porosity follows from the property of plastic incompressibility of the matrix

$$
\dot{f}=(1-f) \operatorname{tr} \mathbf{D}^{p}
$$

The evolution law for the void aspect ratio is determined using an approximate method by assuming that the void shape remains spheroidal upon deformation. The proposed evolution law for the void shape is given by

$$
\frac{\dot{w}}{w}=\frac{3}{2} D_{33}^{p^{\prime}}+\left(\frac{1-3 \alpha_{1}}{f}+3 \alpha_{2}-1\right) \operatorname{tr} \mathbf{D}^{p}
$$

where $\mathbf{D}^{p^{\prime}}=\mathbf{D}^{p}-\frac{1}{3}\left(\operatorname{tr} \mathbf{D}^{p}\right) \mathbf{1}$ denotes the deviatoric part of the plastic deformation rate.

Apart from the evolution laws for $f$ and $w$, an equation for the spin of the void axis $\underline{e}_{3}$ is also required to complete the model. An equation for the same based on the micromechanical analysis of Kailasam and Ponte Castaneda [22] was proposed in Keralavarma and Benzerga [24]. This is omitted here, since all the numerical results presented in this paper correspond to special cases of loading where the rotation of the void axis is prevented.

\section{Numerical Upper-Bound Yield Criterion}

In this section, we present a numerical scheme for computing rigorous upper-bounds to the macroscopic yield loci for RVEs of the type illustrated in Fig. 1, i.e. thick spheroidal shells made of a Hill material and containing a confocal spheroidal void. We generalize the numerical method developed by Gologanu et al. [15] for spheroidal voids in a Von Mises matrix to the more general case of Hill orthotropic matrices. As in Gologanu et al. [15], we employ a large number of velocity fields derived from the family of axisymmetric incompressible velocity fields proposed by Lee and Mear [29] to describe the microscopic velocity field in the RVE. In addition, a homogeneous non-axisymmetric deformation field 
is incorporated so that the method can be applied to the case of orthotropic materials that do not respect transverse isotropy about the void axis. While rigorous upper-bound yield loci are obtained in all cases, the method has the property that the loci will be quasi-exact in the special case of materials that are transversely isotropic about the void axis ${ }^{1}$. For general orthotropic materials, an alternative limit analysis approach that does not require choice of trial velocity fields a priori has recently been proposed by Morin et al. [35]. The method consists of discretising the RVE and using the finite element method to compute the plastic limit load by directly applying a single large load step, without geometry update, such that the limit load is reached in a few iterations. Exact microscopic velocity fields are obtained numerically using this approach, which can potentially yield more accurate results for non-transversely isotropic materials. On the other hand, the velocity fields used in the derivation of the analytical model of Keralavarma and Benzerga [24] is a small subset of the trial velocity fields used in the present limit analysis method and is sufficient for the purpose of evaluation of the analytical model and the approximations therein. The formulation here is restricted to the case of axi-symmetric loading about the void axis, i.e. stress states of the form $\Sigma=\Sigma_{11}\left(\underline{e}_{1} \otimes \underline{e}_{1}+\underline{e}_{2} \otimes \underline{e}_{2}\right)+\Sigma_{33} \underline{e}_{3} \otimes \underline{e}_{3}$, so that the resulting yield locus represents the intersection in stress space of the yield surface with the plane of axisymmetric loading. For simplicity, we further restrict the analysis to the case where the void axis coincides with one of the axes of material orthotropy, although more general cases can be considered by a straightforward extension of the present formulation. In the following derivations, the Cartesian frame $\left(\underline{e}_{1}, \underline{e}_{2}, \underline{e}_{3}\right)$ of Fig. 1 is taken to coincide with the axes of orthotropy of the matrix.

\subsection{Variational definition of the yield locus}

In the kinematic approach of Hill-Mandel homogenization theory, the RVE of Fig. 1 is subjected to homogeneous deformation rate boundary conditions $\underline{v}(\underline{x})=\mathbf{D} \cdot \underline{x}$ on $\partial \Omega$. For a rigid plastic material, the macroscopic plastic dissipation in the RVE, $\Sigma: \mathbf{D} \equiv \Pi(\mathbf{D})$, equals the volume average of the microscopic plastic dissipation according to the HillMandel lemma; i.e. $\boldsymbol{\Sigma}: \mathbf{D}=\langle\boldsymbol{\sigma}: \mathbf{d}\rangle_{\Omega}$. This fact is used to arrive at a variational expression for $\Pi(\mathbf{D})$ as

$$
\Pi(\mathbf{D})=\inf _{\mathbf{d} \in \mathcal{K}(\mathbf{D})}\langle\pi(\mathbf{d})\rangle_{\Omega}
$$

where $\mathcal{K}(\mathbf{D})$ is the set of all incompressible microscopic deformation fields $\mathbf{d}$ that are kinematically admissible with respect to the homogeneous deformation rate $\mathbf{D}$ at the RVE boundary

$$
\mathcal{K}(\mathbf{D})=\left\{\mathbf{d} \mid \exists \underline{v}, \forall \underline{x} \in \Omega, \mathbf{d}=\frac{1}{2}\left(\nabla \underline{v}+\nabla \underline{v}^{T}\right), \operatorname{tr} \mathbf{d}=0 \text { and } \forall \underline{x} \in \partial \Omega, \underline{v}=\mathbf{D} . \underline{x}\right\}
$$

$\underline{v}$ is the trial velocity field and $\pi(\mathbf{d})$ is the microscopic plastic dissipation. The latter is obtained from the maximum rate of dissipation principle as

$$
\pi(\mathbf{d})=\sup _{\boldsymbol{\sigma}^{*} \in \mathcal{C}} \boldsymbol{\sigma}^{*}: \mathbf{d}
$$

\footnotetext{
${ }^{1}$ We assume that the Lee and Mear [29] fields represent the complete family of incompressible axisymmetric velocity fields
} 
where $\mathcal{C}$ denotes the microscopic convex of rigidity. For a Hill orthotropic material, the above evaluates to

$$
\pi(\mathbf{d})=\sigma_{0} d_{e q}, \quad d_{e q} \equiv \sqrt{\frac{2}{3} \mathbf{d}: \hat{\mathbf{h}}: \mathbf{d}}
$$

where $d_{e q}$ is an effective strain rate work conjugate to the reference yield stress $\sigma_{0}$.

The macroscopic convex of reversibility or the region of potentially supportable stresses $\Sigma$ in stress space is given by the inequality

$$
\Sigma: \mathbf{D} \leq \Pi(\mathbf{D}) \quad \forall \mathbf{D}
$$

The macroscopic yield surface is the boundary of the above region defined as the envelope of hyperplanes in stress space, $\boldsymbol{\Sigma}: \mathbf{D}=\Pi(\mathbf{D})$, parameterized by $\mathbf{D}$ [44]. Since $\Pi(\mathbf{D})$ is a homogeneous function of degree one in the components of $\mathbf{D}$, the five ratios of these components may be eliminated between the six equations above to obtain the explicit equation for the macroscopic yield surface. Considering the special case of axisymmetric loading about the void axis, one can see that the macroscopic stress and rate of deformation tensors must be of the form

$$
\begin{gathered}
\boldsymbol{\Sigma}=\Sigma_{11}\left(\underline{e}_{1} \otimes \underline{e}_{1}+\underline{e}_{2} \otimes \underline{e}_{2}\right)+\Sigma_{33} \underline{e}_{3} \otimes \underline{e}_{3}, \\
\mathbf{D}=D_{11} \underline{e}_{1} \otimes \underline{e}_{1}+D_{22} \underline{e}_{2} \otimes \underline{e}_{2}+D_{33} \underline{e}_{3} \otimes \underline{e}_{3}
\end{gathered}
$$

under the assumption that the triad $\left(\underline{e}_{1}, \underline{e}_{2}, \underline{e}_{3}\right)$ coincides with the principal directions of matrix orthotropy. Using (17) and (18) in (16), the region of allowable stresses may be written as

$$
\Sigma_{11}\left(D_{11}+D_{22}+X D_{33}\right) \leq \Pi\left(D_{11}, D_{22}, D_{33}\right) \quad \forall D_{11}, D_{22}, D_{33}, \quad X \equiv \frac{\Sigma_{33}}{\Sigma_{11}}
$$

where $X$ is the ratio of the principal stresses, and the notation $\Pi$ is retained for convenience. For axisymmetric loading, $X$ is related to the stress triaxiality, $T$, by

$$
X=\frac{9 T}{3 T-1}-2, \quad T \equiv \frac{\operatorname{tr} \boldsymbol{\Sigma}}{3 \sqrt{\frac{3}{2} \Sigma: \Sigma}}
$$

In other words, the yield point for an axisymmetric stress state defined by the ratio of the principal stresses $X$ may be obtained by minimizing the right-hand side of equation $(19)_{1}$ with respect to arbitrary values of $D_{i j}$ and using the equality condition in $(19)_{1}$ to solve for $\Sigma_{11}$. However, since $\Pi(\mathbf{D})$ is homogeneous of degree one in $\mathbf{D}$, inequality $(19)_{1}$ is homogeneous in $\mathbf{D}$ and one may scale the components of $\mathbf{D}$ arbitrarily. For convenience we impose the condition

$$
D_{11}+D_{22}+X D_{33}=1
$$

so that we have

$$
\Sigma_{11}=\bar{\Pi}(X) \equiv \inf _{D_{11}, D_{22}, D_{33}} \Pi\left(D_{11}, D_{22}, D_{33}\right)
$$

subject to the constraint (21). Note that the choice of constraint (21) implies that the solution for $\Sigma_{11}$ must be positive. However, since the yield surface is centrosymmetric about the origin, $-\Sigma_{11}$ is also a solution. $\Pi\left(D_{11}, D_{22}, D_{33}\right)$ is in turn calculated by evaluation of the infimum in equation (12) using a finite set of kinematically admissible trial velocity fields as defined by (13). 


\subsection{Trial velocity fields}

The macroscopic deformation tensor in (18) may be written as a linear combination of an axisymmetric deformation rate, $\mathbf{D}^{a}$, and a pure shear deformation in the transverse plane of the RVE, $\mathbf{D}^{s}$, as

$$
\begin{gathered}
\mathbf{D}=\mathbf{D}^{a}+\mathbf{D}^{s} \\
\mathbf{D}^{a}=D_{11}^{a}\left(\underline{e}_{1} \otimes \underline{e}_{1}+\underline{e}_{2} \otimes \underline{e}_{2}\right)+D_{33}^{a} \underline{e}_{3} \otimes \underline{e}_{3}, \quad \mathbf{D}^{s}=D_{11}^{s}\left(\underline{e}_{1} \otimes \underline{e}_{1}-\underline{e}_{2} \otimes \underline{e}_{2}\right)
\end{gathered}
$$

where

$$
D_{11}^{a}=\frac{D_{11}+D_{22}}{2}, \quad D_{33}^{a}=D_{33}, \quad D_{11}^{s}=\frac{D_{11}-D_{22}}{2}
$$

For the numerical evaluation of $\Pi\left(D_{11}, D_{22}, D_{33}\right)$ in (22), using (12), we consider trial velocity fields of the form

$$
\begin{aligned}
& \underline{v}=\underline{v}^{a}+\mathbf{D}^{s} \cdot \underline{x} \\
& \mathbf{d}=\frac{1}{2}\left(\nabla \underline{v}+\nabla \underline{v}^{T}\right)=\mathbf{d}^{a}+\mathbf{D}^{s}
\end{aligned}
$$

where the first component, $\underline{v}^{a}$, is an axisymmetric velocity field and the second velocity field corresponds to a homogeneous pure shear deformation, $\mathbf{D}^{s}$, in the transverse plane of the RVE. From the kinematic boundary conditions, equation (13), we obtain

$$
\underline{v}=\mathbf{D} \cdot \underline{x} \quad \Rightarrow \quad \underline{v}^{a}=\mathbf{D}^{a} \cdot \underline{x} \quad \text { on } \quad \partial \Omega
$$

We choose the field $\underline{v}^{a}$ from the family of incompressible axisymmetric velocity fields proposed by Lee and Mear [29], which is assumed to represent the complete set of such velocity fields. Due to the spheroidal geometry of the problem, we choose to work with the spheroidal coordinate system $(\lambda, \beta, \varphi)$, whose base vectors are defined in the Cartesian frame of Fig. 1 as

$$
\begin{array}{r}
\underline{e}_{\lambda}=\left\{a \sin \beta \cos \varphi \underline{e}_{1}+a \sin \beta \sin \varphi \underline{e}_{2}+b \cos \beta \underline{e}_{3}\right\} / \sqrt{g_{\lambda \lambda}} \\
\underline{e}_{\beta}=\left\{b \cos \beta \cos \varphi \underline{e}_{1}+b \cos \beta \sin \varphi \underline{e}_{2}-a \sin \beta \underline{e}_{3}\right\} / \sqrt{g_{\lambda \lambda}} \\
\underline{e}_{\varphi}=-\sin \varphi \underline{e}_{1}+\cos \varphi \underline{e}_{2} \\
g_{\lambda \lambda} \equiv a^{2} \sin ^{2} \beta+b^{2} \cos ^{2} \beta, \quad\left\{\begin{array}{l}
a=c \cosh \lambda, b=c \sinh \lambda \\
a=c \sinh \lambda, b=c \cosh \lambda
\end{array}\right.
\end{array}
$$

where $c$ denotes the semi-focal length of the spheroidal void. In the spheroidal system, iso- $\lambda$ surfaces are confocal spheroids with focal length, $2 c$, so that the surfaces of the void and the RVE correspond to constant values of $\lambda$, designated $\lambda_{1}$ and $\lambda_{2}$ respectively. The eccentricity, $e$, of the current confocal spheroid is related to $\lambda$ as $e=1 / \cosh \lambda$, with $e_{1}$ and $e_{2}$ denoting the eccentricities of the void and the RVE respectively. The components 
of the Lee and Mear [29] velocity fields, expressed in spheroidal coordinates, are

$$
\begin{aligned}
& \left\{\begin{array}{l}
v_{\lambda}^{a}(\lambda, \beta)=c^{2} / \sqrt{g_{\lambda \lambda}}\left\{B_{00} / \sinh (\lambda)\right. \\
\left.+\sum_{k=2,4, . .}^{+\infty} \sum_{m=0}^{+\infty} k(k+1)\left[B_{k m} Q_{m}^{1}(w)+C_{k m} P_{m}^{1}(w)\right] P_{k}(u)\right\} \\
v_{\beta}^{a}(\lambda, \beta)=c^{2} / \sqrt{g_{\lambda \lambda}}\left\{\sum _ { k = 2 , 4 , . . } ^ { + \infty } \sum _ { m = 1 } ^ { + \infty } m ( m + 1 ) \left[B_{k m} Q_{m}(w)\right.\right. \\
\left.\left.+C_{k m} P_{m}(w)\right] P_{k}^{1}(u)\right\}
\end{array}\right. \\
& \left\{\begin{array}{c}
\left.v_{\lambda}^{a}(\lambda, \beta)=c^{2} / \sqrt{g_{\lambda \lambda}}\left\{B_{00} / \cosh (\lambda)+C_{k m} P_{m}^{1}(w)\right] P_{k}(u)\right\} \\
+\sum_{k=2,4, . .}^{+\infty} \sum_{m=0}^{+\infty} k(k+1) i^{m}\left[i B_{k m} Q_{m}^{1}(w)+i^{m}\left[i B_{k m} Q_{m}(w)\right.\right. \\
v_{\beta}^{a}(\lambda, \beta)=c^{2} / \sqrt{g_{\lambda \lambda}}\left\{\sum_{k=2,4, . .}^{+\infty} \sum_{m=1}^{+\infty} m\left(m+C_{k m} P_{m}(w)\right] P_{k}^{1}(u)\right\}
\end{array}\right.
\end{aligned}
$$

where

$$
w \equiv\left\{\begin{array}{ll}
\cosh \lambda & (\mathrm{p}) \\
i \sinh \lambda & (\mathrm{o})
\end{array} ; \quad u \equiv \cos \beta\right.
$$

In the above expressions, $P_{n}^{m}$ and $Q_{n}^{m}$ represent associated Legendre functions of the first and second kinds respectively, of order $m$ and degree $n$ [17], and $B_{k m}$ and $C_{k m}$ are arbitrary real constants. The requirement of homogeneous boundary strain rate, equation $(27)_{2}$ for some unspecified $\mathbf{D}^{a}$, leads to the following expressions for $D_{11}^{a}$ and $D_{33}^{a}$ in terms of the coefficients $B_{k m}$ and $C_{k m}$ of the Lee-Mear fields

$$
D_{11}^{a}=\frac{3 c^{2}}{a_{2}^{2}-b_{2}^{2}}\left[G_{2}\left(\lambda_{2}\right)-\frac{3 a_{2}}{b_{2}} F_{2}\left(\lambda_{2}\right)\right], D_{33}^{a}=\frac{3 c^{2}}{a_{2}^{2}-b_{2}^{2}}\left[G_{2}\left(\lambda_{2}\right)-\frac{3 b_{2}}{a_{2}} F_{2}\left(\lambda_{2}\right)\right]
$$

and linear constraints on the remaining coefficients $B_{k m}$ and $C_{k m}$, given by [see 15]

$$
\begin{aligned}
& \left\{\begin{array}{l}
e_{2}^{3} B_{00} /\left(3\left(1-e_{2}^{2}\right)\right)+\left(3-e_{2}^{2}\right) F_{2}\left(\lambda_{2}\right) / \sqrt{1-e_{2}^{2}}-G_{2}\left(\lambda_{2}\right)=0 \\
-e_{2}^{3} B_{00} /\left(3 \sqrt{1-e_{2}^{2}}\right)+\left(3-2 e_{2}^{2}\right) F_{2}\left(\lambda_{2}\right) / \sqrt{1-e_{2}^{2}}-G_{2}\left(\lambda_{2}\right)=0
\end{array}\right. \\
& F_{k}\left(\lambda_{2}\right)=G_{k}\left(\lambda_{2}\right)=0, \quad k=4,6,8 \ldots
\end{aligned}
$$

where

$$
\begin{aligned}
& \left\{\begin{array}{l}
F_{k}(\lambda) \equiv \sum_{m=0}^{+\infty}\left[B_{k m} Q_{m}^{1}(w)+C_{k m} P_{m}^{1}(w)\right] \\
G_{k}(\lambda) \equiv \sum_{m=1}^{+\infty} m(m+1)\left[B_{k m} Q_{m}(w)+C_{k m} P_{m}(w)\right]
\end{array}\right. \\
& \left\{\begin{array}{l}
F_{k}(\lambda) \equiv \sum_{m=0}^{+\infty} i^{m}\left[i B_{k m} Q_{m}^{1}(w)+C_{k m} P_{m}^{1}(w)\right] \\
G_{k}(\lambda) \equiv \sum_{m=1}^{+\infty} m(m+1) i^{m}\left[i B_{k m} Q_{m}(w)+C_{k m} P_{m}(w)\right]
\end{array}\right.
\end{aligned}
$$


Note that equation (34) is obtained by using equations (33) to eliminate the components of $\mathbf{D}^{a}$ from the condition $\operatorname{tr} \mathbf{D}^{a}=3 c^{3} B_{00} /\left(a_{2} b_{2}^{2}\right)$. The constraint of equation (21) may now be written using spheroidal coordinates by using (33) and (24) in (21) as

$$
\frac{3 c^{2}}{a_{2}^{2}-b_{2}^{2}}\left[(2+X) G_{2}\left(\lambda_{2}\right)-3\left(2 \frac{a_{2}}{b_{2}}+X \frac{b_{2}}{a_{2}}\right) F_{2}\left(\lambda_{2}\right)\right]=1
$$

Thus, the numerical evaluation of the yield point under axisymmetric loading (17), specified by the stress ratio $X=\Sigma_{33} / \Sigma_{11}$, corresponds to minimization of the function $\bar{\Pi}(X)$ in (22) using trial velocity fields of the form (26), subject to linear constraints on the values of the coefficients $B_{k m}$ and $C_{k m}$, given by equations (34), (35) and (37).

\subsection{Numerical minimization of the plastic dissipation}

For given values of the microstructural parameters $f$ and $w$, the eccentricities of the void and RVE may be obtained from equation (6). The semi-focal length, $c$, may be chosen arbitrarily since the problem is scale invariant. Combining (12) and (15), we may write the macroscopic plastic dissipation as

$$
\Pi(\mathbf{D})=\inf _{\mathbf{d} \in \mathcal{K}(\mathbf{D})} \sigma_{0}\left\langle d_{e q}\right\rangle_{\Omega}, \quad d_{e q}=\sqrt{\frac{2}{3} \mathbf{d}: \hat{\mathrm{h}}: \mathbf{d}}
$$

$\mathbf{d}$ is computed using the decomposition of the microscopic velocity field, $\underline{v}$, in (26), where $\underline{v}^{a}$ is chosen to be a finite sub-set of the infinite double-series of the Lee-Mear fields in equations (30)-(31). Adopting the velocity fields corresponding to the coefficients $B_{00}, B_{k m}$ and $C_{k m}$, where $k=2,4,6, . ., K$ and $m=0,1,2, . ., M$, we have a total of $N=K(M+1)+2$ trial velocity fields (including the homogeneous pure shear field $\mathbf{D}^{s} \cdot \underline{x}$ ). It is clear that $\mathbf{d}$ is a homogeneous linear function of the coefficients $B_{00}, B_{k m}, C_{k m}$ and the in-plane shear strain rate $D_{11}^{s}$. Let $[\mathbf{d}]$ stand for the $6 \times 1$ Voigt vector representation of $\mathbf{d}$ in the frame $\left(\underline{e}_{1}, \underline{e}_{2}, \underline{e}_{3}\right)$. We may write

$$
[\mathbf{d}]=[\mathcal{L}(\lambda, \beta, \varphi)][A]
$$

where $[\mathcal{L}(\lambda, \beta, \varphi)]$ is a $6 \times N$ matrix whose components are functions of the coordinates $(\lambda, \beta, \varphi)$, and $[A]$ is a $N \times 1$ column vector defined as

$$
\left.[A] \equiv\left[\begin{array}{lll}
B_{00} & {\left[B_{k m}\right.}
\end{array}\right]^{T} \quad\left[C_{k m}\right]^{T} \quad D_{11}^{s}\right]^{T}
$$

In (40), $\left[B_{k m}\right]$ and $\left[C_{k m}\right]$ denote column vectors of the corresponding Lee-Mear coefficients. The expression for $d_{e q}$ may be written as

$$
d_{e q}=\sqrt{\frac{2}{3}[A]^{T}[\mathcal{L}]^{T}[\hat{\mathrm{h}}][\mathcal{L}][A]}=\sqrt{[A]^{T}[\mathcal{M}][A]}, \quad[\mathcal{M}] \equiv \frac{2}{3}[\mathcal{L}]^{T}[\hat{\mathrm{h}}][\mathcal{L}]
$$

where $[\hat{\mathrm{h}}]$ is the coordinatization of the anisotropy tensor, $\hat{\mathrm{h}}$, in the frame $\left(\underline{e}_{1}, \underline{e}_{2}, \underline{e}_{3}\right)$, expressed in Voigt form. Since $\left(\underline{e}_{1}, \underline{e}_{2}, \underline{e}_{3}\right)$ is taken to be the frame of material orthotropy, [ $[\hat{\mathrm{h}}]$ will be a diagonal $6 \times 6$ matrix. Combining equations (22), (38) and (41), the optimization 
problem for determination of the yield point under axisymmetric loading specified by the stress ratio $X$ may now be written in the form

$$
\bar{\Pi}(X)=\inf _{[A] \in \mathcal{R}^{N}} \sigma_{0}\left\langle\sqrt{[A]^{T}[\mathcal{M}][A]}\right\rangle_{\Omega}
$$

However, the components of $[A]$ are subject to the constraints given by equations (34), (35) and (37). These make a total of $K$ linear equality constraints, which may be used to eliminate $K$ of the $N$ unknowns as

$$
[A]=[\mathcal{C}][B]+\left[A_{0}\right]
$$

where $[B]$ is an $\bar{N} \times 1$ vector of the remaining independent unknowns $\bar{N}=N-K,[\mathcal{C}]$ is a constraint matrix of dimensions $N \times \bar{N}$ and $\left[A_{0}\right]$ is a constant vector. Using (43) in (42), we have

$$
\bar{\Pi}(X)=\inf _{[B] \in \mathcal{R}^{\bar{N}}} \tilde{\Pi}([B]), \quad \tilde{\Pi}([B]) \equiv \sigma_{0}\left\langle\sqrt{\left([\mathcal{C}][B]+\left[A_{0}\right]\right)^{T}[\mathcal{M}]\left([\mathcal{C}][B]+\left[A_{0}\right]\right)}\right\rangle_{\Omega}
$$

The above represents an unconstrained minimization problem where $\tilde{\Pi}([B])$ is the objective function in $\bar{N}$ variables, $[B]$, and the space of admissible values of $[B]$ is $\mathcal{R}^{\bar{N}}$. Existence of the minimum is guaranteed by the fact that $[\hat{\mathrm{h}}]$ is a positive definite matrix, which implies that $[\mathcal{M}]$ is positive semi-definite and hence the objective function is convex. Further, the minimum value of the objective function (and therefore the yield point according to (22)) will be unique even though the minimum may be attained for an infinite number of values of $[B]$. The Jacobian of the objective function is given by

$$
\frac{\partial \tilde{\Pi}}{\partial[B]}=\sigma_{0}\left\langle\frac{[\mathcal{C}]^{T}[\mathcal{M}]\left([\mathcal{C}][B]+\left[A_{0}\right]\right)}{\sqrt{\left([B]^{T}[\mathcal{C}]^{T}+\left[A_{0}\right]^{T}\right)[\mathcal{M}]\left([\mathcal{C}][B]+\left[A_{0}\right]\right)}}\right\rangle_{\Omega}
$$

The above problem can be solved for various values of the macroscopic stress triaxiality, $T$, to obtain individual points on the yield locus using equation (22). A program is developed to perform the above minimization using a conjugate gradient minimization algorithm [12]. Henceforth, the yield locus obtained using the above method will be referred to as the 'exact numerical yield locus'. The analytical criterion of section 2 is compared with the exact numerical yield loci for various proportional loading paths, void shapes, volume fractions and material anisotropy coefficients in the following section.

\section{Results}

\subsection{Yield Criterion}

The numerical method of the previous section can be used to generate the yield locus for axisymmetric loading about the void axis, i.e. stress states of the form $\Sigma=\Sigma_{11}\left(\underline{e}_{1} \otimes\right.$

$\left.\underline{e}_{1}+\underline{e}_{2} \otimes \underline{e}_{2}\right)+\Sigma_{33} \underline{e}_{3} \otimes \underline{e}_{3}$. The analytical equation of the yield locus (2) for axisymmetric loading reads

$$
\frac{h_{q}^{\prime} C}{\sigma_{0}^{2}}\left(\Sigma_{33}-\Sigma_{11}+\frac{3}{2} \eta \Sigma_{h}\right)^{2}+2(g+1)(g+f) \cosh \left(\kappa \frac{\Sigma_{h}}{\sigma_{0}}\right)-(g+1)^{2}-(g+f)^{2}=0
$$


Table 1: Table of material anisotropy parameters used in the numerical computations.

\begin{tabular}{lcccccc}
\hline Name & $h_{1}$ & $h_{2}$ & $h_{3}$ & $h_{4}$ & $h_{5}$ & $h_{6}$ \\
\hline Isotropic & 1.000 & 1.000 & 1.000 & 1.000 & 1.000 & 1.000 \\
\hline Material 1 & 1.000 & 1.000 & 1.000 & 2.333 & 2.333 & 1.000 \\
\hline Material 2 & 1.000 & 1.000 & 1.000 & 0.500 & 0.500 & 1.000 \\
\hline Material 3 & 1.650 & 0.778 & 0.893 & 1.378 & 0.943 & 1.627 \\
\hline
\end{tabular}

where $\Sigma_{h}$ is defined by $\Sigma_{h} \equiv \boldsymbol{\Sigma}: \mathbf{X}=2 \alpha_{2} \Sigma_{11}+\left(1-2 \alpha_{2}\right) \Sigma_{33}$ and $h_{q}^{\prime}$ is defined similar to $h_{q}$ in equation (8) using the components of the tensor $\mathbb{h}$ instead of $\hat{\mathrm{h}}$. The criterion also depends on the anisotropy factors $h, h_{q}$ and $h_{t}$ defined in (8). In the case when $\left(\underline{e}_{1}, \underline{e}_{2}, \underline{e}_{3}\right)$ coincides with the axes of orthotropy, the Voigt form of the tensors $\mathfrak{h}$ and $\hat{\mathfrak{h}}$ are diagonalized and they take on simplified expressions

$$
\begin{gathered}
h=2\left[\frac{2\left(\hat{h}_{1}+\hat{h}_{2}+\hat{h}_{3}\right)+3\left(\hat{h}_{4}+\hat{h}_{5}+\hat{h}_{6}\right)}{15}\right]^{1 / 2}, \quad h_{t}=h^{2}-\frac{3}{4}\left(\hat{h}_{1}+\hat{h}_{2}+2 \hat{h}_{6}\right) \\
h_{q}=\frac{\hat{h}_{1}+\hat{h}_{2}+4 \hat{h}_{3}}{6}, \quad h_{q}^{\prime}=\frac{h_{1}+h_{2}+4 h_{3}}{6}
\end{gathered}
$$

In the above expressions, $h_{i}$ and $\hat{h}_{i}(i=1 . .6)$ represent the diagonal elements of the Voigt matrix representation of the tensors $\mathrm{h}$ and $\hat{\mathrm{h}}$ respectively. These are related by [see 5]

$$
\begin{gathered}
\hat{h}_{1}=\frac{-h_{1}+2 h_{2}+2 h_{3}}{h_{1} h_{2}+h_{2} h_{3}+h_{3} h_{1}}, \hat{h}_{2}=\frac{2 h_{1}-h_{2}+2 h_{3}}{h_{1} h_{2}+h_{2} h_{3}+h_{3} h_{1}}, \hat{h}_{3}=\frac{2 h_{1}+2 h_{2}-h_{3}}{h_{1} h_{2}+h_{2} h_{3}+h_{3} h_{1}}, \\
\hat{h}_{4}=\frac{1}{h_{4}}, \hat{h}_{5}=\frac{1}{h_{5}}, \hat{h}_{6}=\frac{1}{h_{6}}
\end{gathered}
$$

It may also be noted that, in the case of transverse isotropy about the $\underline{e}_{3}$ axis, $h_{q}^{\prime}=1 / h_{q}$.

A detailed tabulation of the Hill anisotropy coefficients in practically important engineering materials is available in Benzerga [2]. For the numerical results presented here, we have chosen to work with a set of fictitious material parameters that are loosely based on the observed values of the Hill coefficients in engineering materials. Table 1 shows the values of the Hill coefficients for four different materials. The first row corresponds to an isotropic material, which is used as a reference. Materials 1 and 2 are transversely isotropic materials with properties similar to those analyzed in Benzerga and Besson [5]. The properties of Material 1 are similar to those observed in thick Al sheets. Material 3 is not transversely isotropic and the properties are based on experimentally determined values for C.P. grade Titanium ${ }^{2}$. In particular, the chosen material designations and properties are identical to those presented in Keralavarma and Benzerga [24] for consistency.

Figs. 2(a)-(d) show the yield loci for the four materials from Table 1 for prolate cavities of aspect ratio, $w=5$, and three different values of the porosity. The loci are plotted with

\footnotetext{
${ }^{2}$ Since the available data in the literature for $\mathrm{Ti}$ correspond to thin sheets [30], for which the out of plane strain ratios are not reported, these we assumed to be unity while calculating the data in Table 1 .
} 


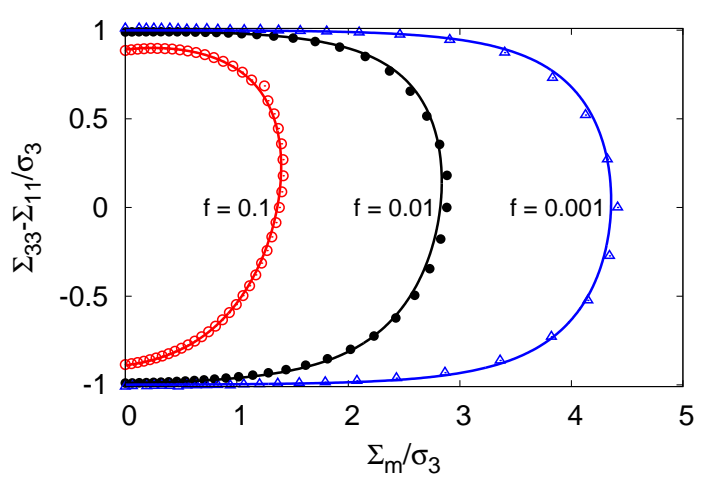

(a)

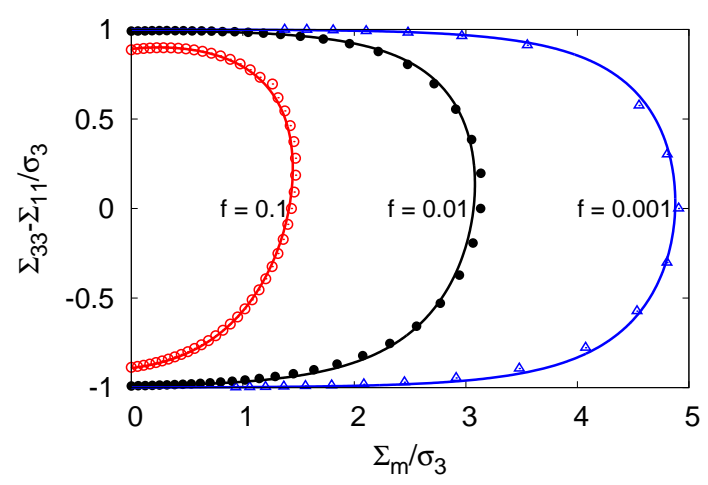

(c)

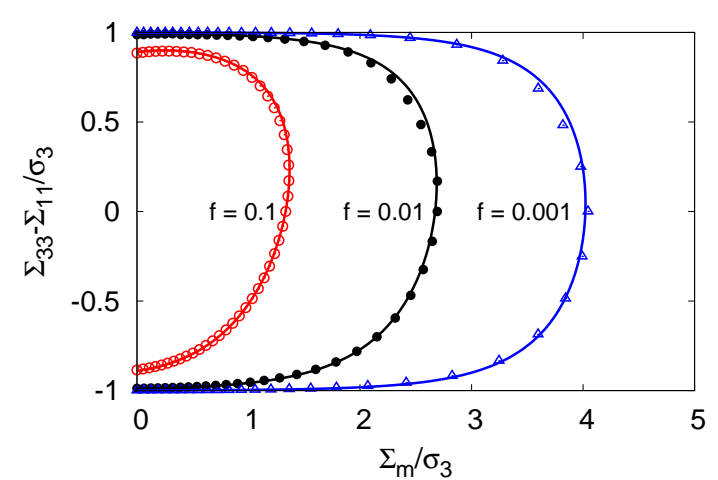

(b)

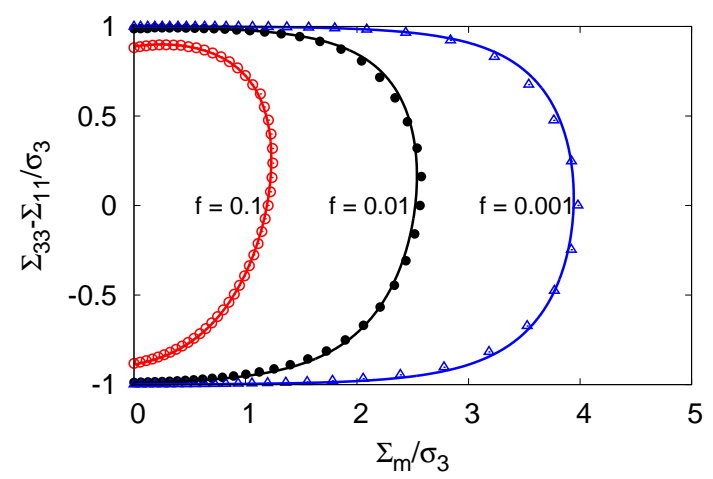

(d)

Figure 2: Comparison of the analytical and numerical yield loci for prolate cavities. (a) Isotropic matrix (b) Material 1 (c) Material 2 (d) Material 3 and three values of the porosity, $f$. In all cases, $w=5$. The solid lines correspond to the analytical criterion of equation (46).

the mean stress $\Sigma_{m}=\frac{1}{3} \Sigma_{k k}$ as the abscissa and $\Sigma_{33}-\Sigma_{11}$ as the ordinate, whose absolute value equals the Von Mises effective stress for axisymmetric loading. The stresses are normalized by $\sigma_{3}$, the yield stress of the matrix in the $\underline{e}_{3}$ direction, which coincides with one of the axes of orthotropy in all the cases considered here. The numerical yield loci are plotted using discrete points, while the continuous lines correspond to the analytical criterion of equation (46). In all the results presented here, the numerical yield loci are calculated using twenty two trial velocity fields corresponding to the Lee-Mear coefficients $B_{00}, B_{k m}, C_{k m}(k=2,4$ and $m=0 . .5)$ and the homogeneous shear strain rate, $D_{11}^{s}$. It is clear from the figure that the analytical criterion provides a close agreement with the upper-bound numerical yield loci (which may be considered quasi-exact for the isotropic matrix and materials 1 and 2, but not material 3) over a wide range of values of the porosity and for all four materials considered. It may also be mentioned that, while the new criterion in the case of prolate cavities and the isotropic matrix differs slightly from that of Gologanu et al. [15] due to the different approach adopted in the definition of the parameter $\kappa$, the loci of Fig. 2(a) are nevertheless in very good agreement with their results.

Similar results for oblate cavities of aspect ratio, $w=1 / 5$, are presented in Figs. 3(a)- 


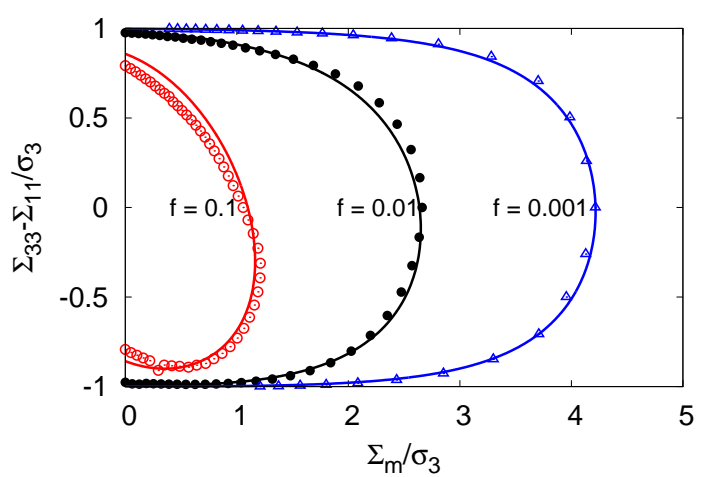

(a)

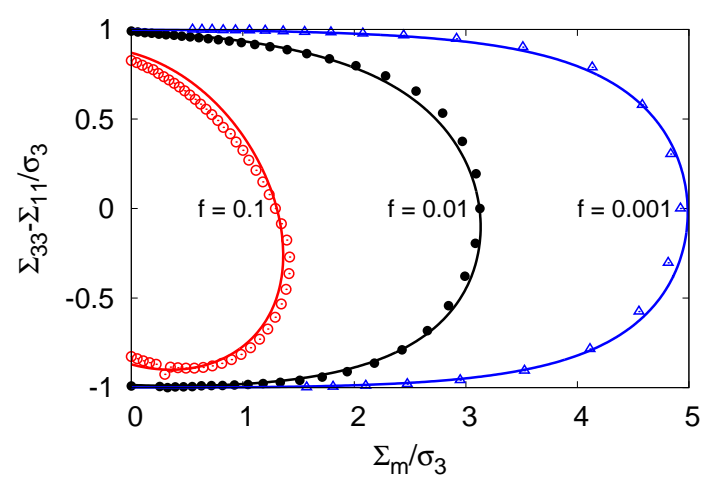

(c)

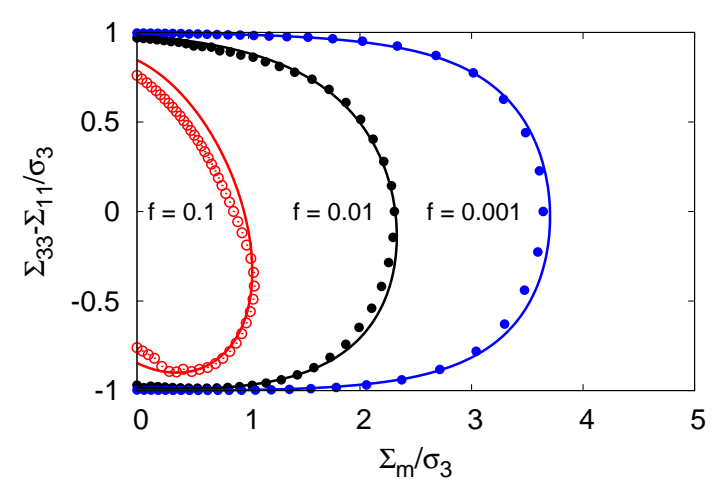

(b)

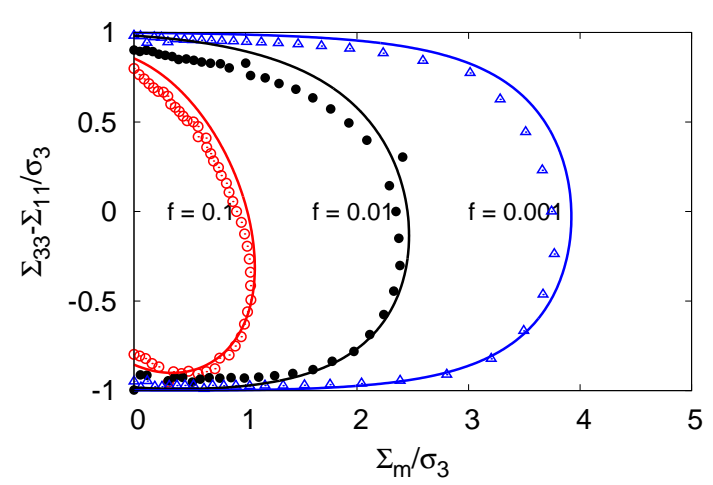

(d)

Figure 3: Comparison of the analytical and numerical yield loci for oblate cavities. (a) Isotropic matrix (b) Material 1 (c) Material 2 (d) Material 3 and three values of the porosity, $f$. In all cases, $w=1 / 5$. The solid lines correspond to the analytical criterion of equation (46).

(d). The new criterion is again seen to agree closely with the numerical data. However, for certain values of the stress triaxiality, the analytical loci are seen to fall slightly inside the numerical yield loci. Since the analytical criterion was derived using a subset of the velocity fields used in computing the numerical locus, this indicates that some of the approximations involved in the derivation of the former could potentially lead to violation of the upper bound character of the solution. However, since the numerical locus is itself a rigorous upper bound to the true locus and the violations observed in Figs. 3(a)-(d) are not significant, it is expected that the analytical solution will be close to the true locus, although upper bound character cannot be guaranteed.

In order to further characterize the yield criterion, we look at the variation of the yield point under axisymmetric loading for two values of the stress triaxiality, $T=1$ and $T=3$, as a function of the void aspect ratio. The former value is representative of the triaxiality in notched tension specimens while the latter value is representative of $T$ in the plastic zone near the tip of sharp cracks. Figs. 4 and 5 show the variation of the hydrostatic stress at yield, designated $\Sigma_{m}^{y}$, as a function of the void aspect ratio for $T=1$ and $T=3$ respectively. Subfigures (a)-(d) correspond the four different material properties from Table 1 . The discrete points represent the numerically determined values 


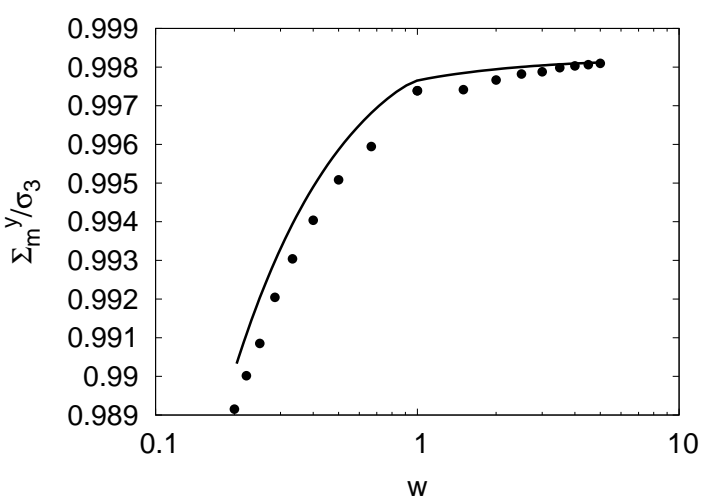

(a)

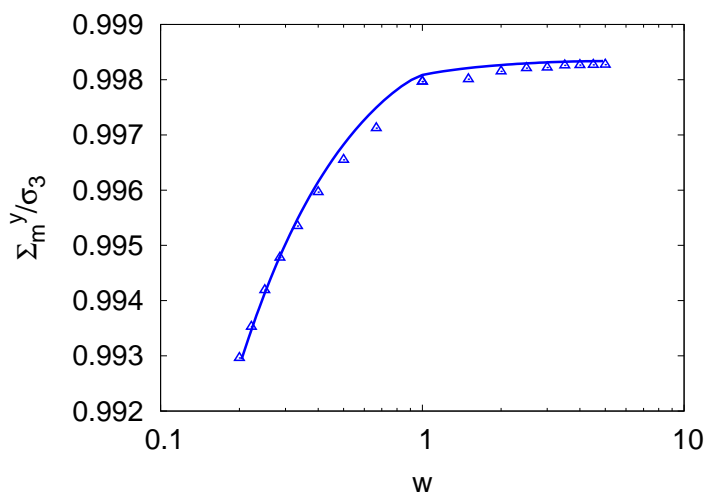

(c)

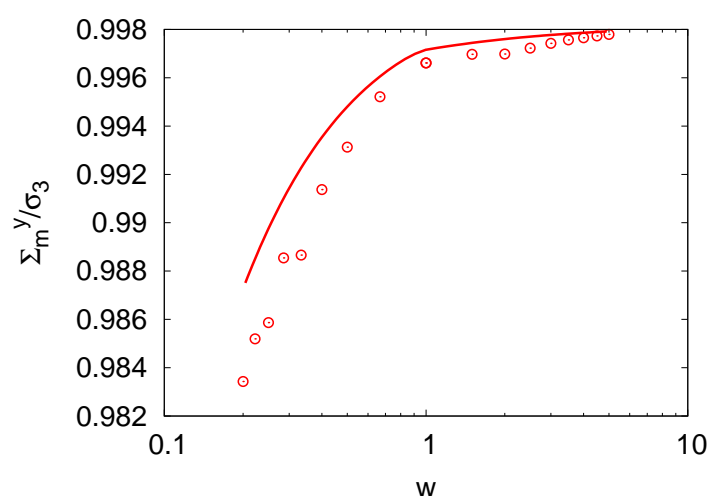

(b)

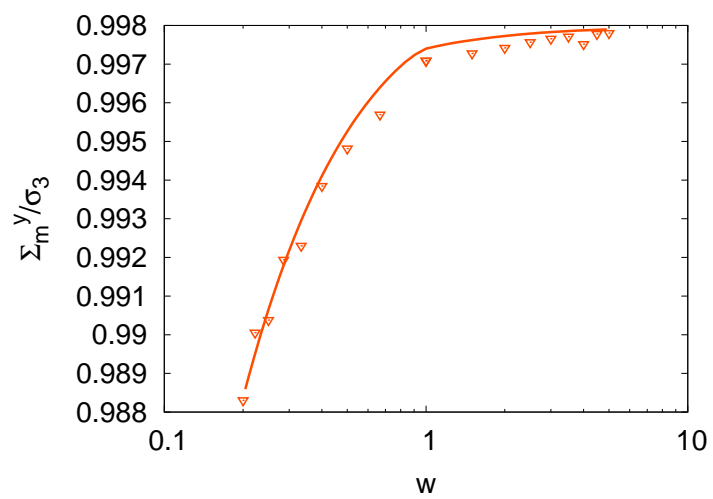

(d)

Figure 4: Variation of the hydrostatic stress at yield, $\Sigma_{m}^{y}$, under proportional axisymmetric loading, as a function of the void aspect ratio, $w$, for porosity $f=0.001$, and stress triaxiality, $T=1$. (a) Isotropic matrix (b) Material 1 (c) Material 2 (d) Material 3. The discrete points are the numerically determined yield points, the solid line correspond to the analytical criterion of equation (46).

and the solid line corresponds to the analytical prediction from equation (46). Again, the analytical predictions are seen to match closely the numerical values for the yield points, which shows that the analytical yield locus matches the exact numerical locus over a wide range of void aspect ratios.

\subsection{Evolution of Porosity}

In general, validation of the microstructure evolution equations require the integration of the constitutive equations for specific loading paths and comparison with predictions from finite-element simulations on porous unit-cells. A preliminary study of this type has been conducted and the results are presented in section 5. However, one may also use the numerical limit-analysis approach to determine the macroscopic dilatancy, $D_{m}$, using the trial velocity fields that minimize the macroscopic plastic dissipation. In the case of transversely isotropic materials, since the Lee-Mear fields are assumed to span the space of axisymmetric velocity fields, the resulting value of $\dot{f}$ may be expected to be close to the true rate of porosity growth.

Fig. 6 illustrate the variation of $D_{m} / D_{m}^{s p h}$ under axisymmetric loading as a function 


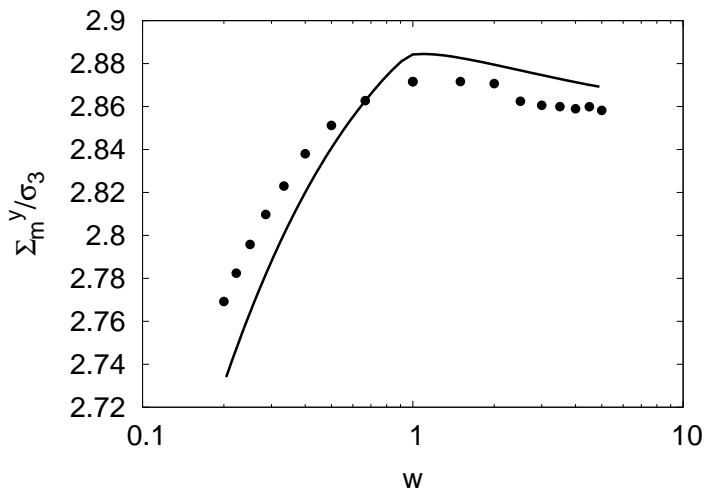

(a)

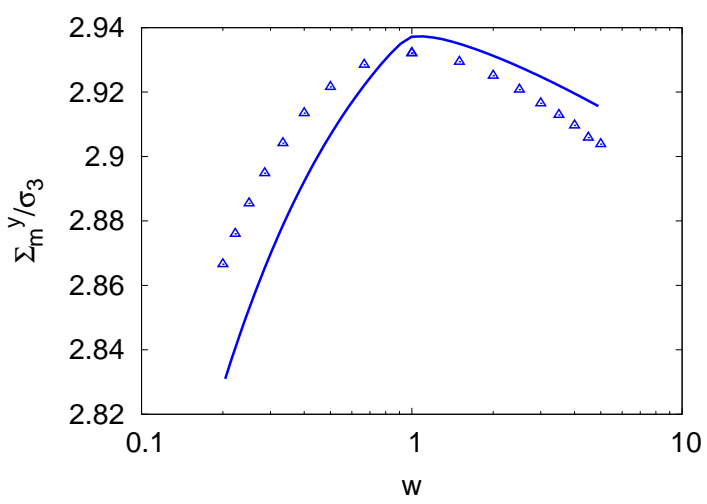

(c)

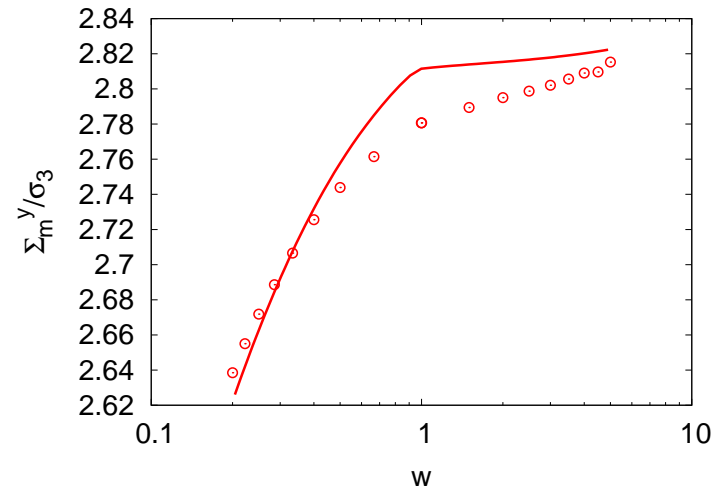

(b)

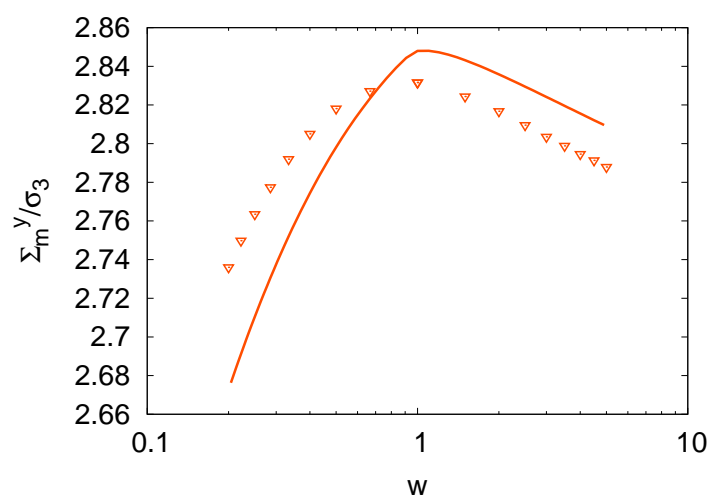

(d)

Figure 5: Variation of the hydrostatic stress at yield, $\Sigma_{m}^{y}$, under proportional axisymmetric loading, as a function of the void aspect ratio, $w$, for porosity $f=0.001$, and stress triaxiality, $T=3$. (a) Isotropic matrix (b) Material 1 (c) Material 2 (d) Material 3. The discrete points are the numerically determined yield points, the solid line correspond to the analytical criterion of equation (46). 


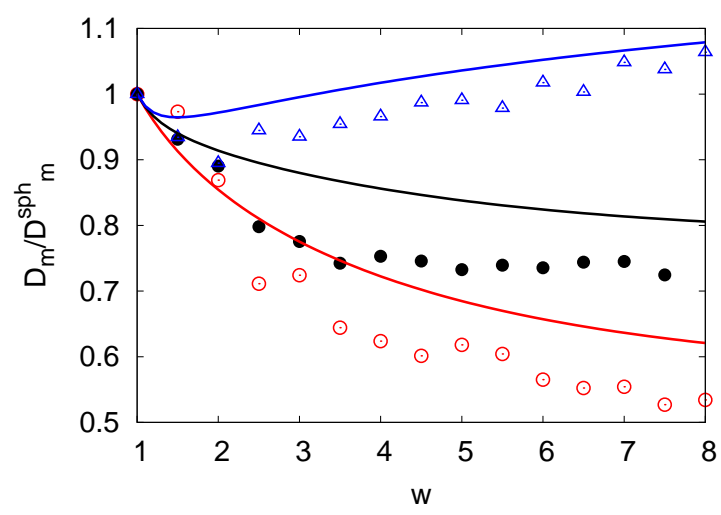

(a)

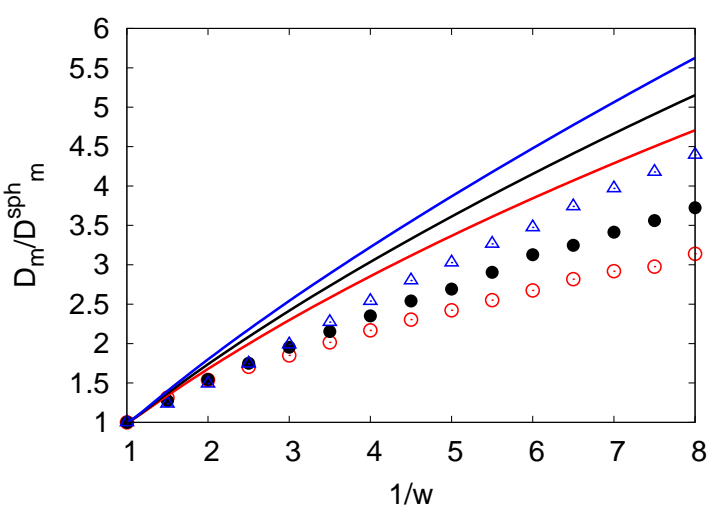

(b)

Figure 6: $D_{m} / D_{m}^{s p h}$ as a function of the void aspect ratio for (a) prolate cavities (b) oblate cavities, stress triaxiality, $T=1$ and porosity, $f=0.01$. The solid line corresponds to the predictions from the analytical model. Discrete points correspond to numerically determined values for Isotropic matrix $(\bullet)$, Material $1(\circ)$ and Material $2(\triangle)$ from Table 1.

of the void aspect ratio, for $f=0.01$, macroscopic stress triaxiality, $T=1$, and three transversely isotropic materials from Table $1 . D_{m}^{s p h}$ here corresponds to the value of $D_{m}$ for a spherical void $(w=1)$. Note that since, in general, the spherical and the non-spherical RVEs have different yield points, and the materials being considered are not hardenable, the comparison is made for the case that the two RVEs have the same axial strain rate, i.e. $D_{33}=D_{33}^{s p h}$. The actual value of $D_{m}^{s p h}$, used in the calculations reported here, is obtained using a void with a vanishingly small eccentricity, which may be considered for all practical purposes to be a sphere. Discrete points in the figure correspond to numerically obtained values of $D_{m} / D_{m}^{s p h}$ using the method described in section 3 . The solid lines correspond to the model predictions using equations (9) and (46). In both the prolate and the oblate cases, the analytical predictions show acceptable agreement with the numerical results for the variation of $D_{m} / D_{m}^{s p h}$ with the void aspect ratio. Since the plastic strain rate is tied to the direction of the normal to the yield locus, this result indicates that not only are the analytical and exact numerical yield loci in good agreement with each other, but also their slopes are closely matched such that good predictions for the plastic strain rate may be expected using the analytical criterion and the associated flow rule.

\section{Finite-Element Simulations}

An alternative approach to validate the evolution equations for $f$ and $w$ is to compare them against direct finite element simulations on porous unit cells. However, a systematic study of this nature will require an extensive investigation involving a large number of test cases, in the spirit of previous works [27, 38, 25] and others. This is especially true in the case of coupled void shape and anisotropy effects, where the parameter space to be explored is large. While such an investigation is beyond the scope of the present work, we present a limited set of finite element calculations for transversely isotropic matrices and axisymmetric states of loading, using the object-oriented finite element program, Zebulon [10]. A cylindrical RVE, made of a transverse isotropic Hill matrix and containing 


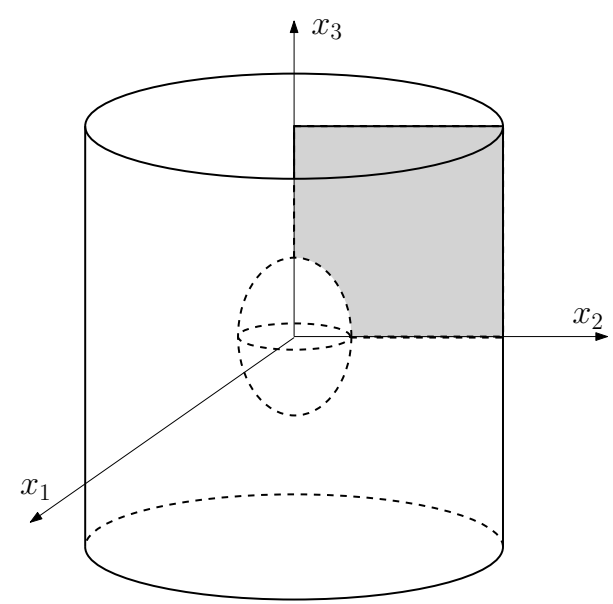

(a)

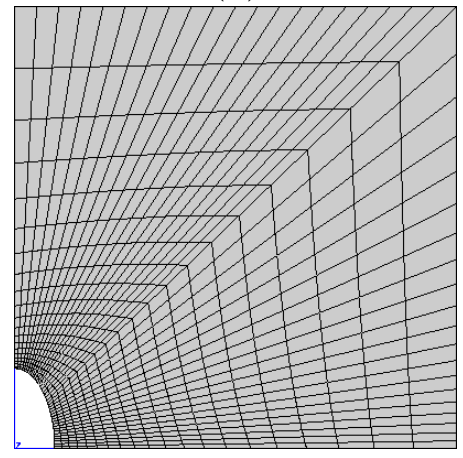

(c)

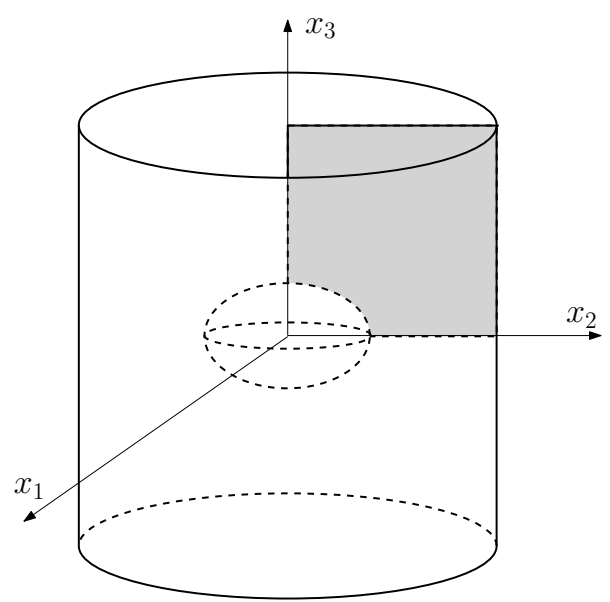

(b)

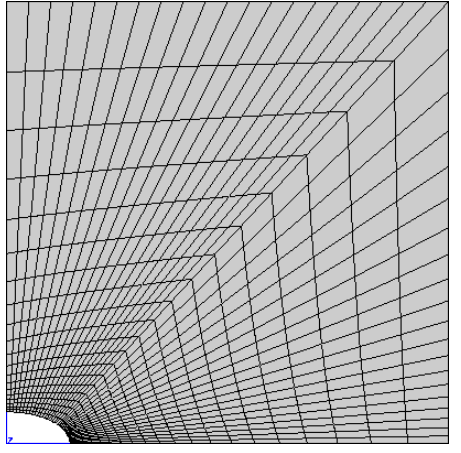

(d)

Figure 7: Sketch of the RVEs used in the finite element unit-cell calculations: (a) prolate void and (b) oblate void. A 2D axisymmetric analysis is performed and symmetry is invoked such that only the shaded region is analyzed. The corresponding finite element meshes are also shown: (c) prolate void $(w=2)$ and (d) oblate void $(w=1 / 2)$. Porosity $f=0.001$ for both cases.

a spheroidal void in the center (Figs. 7(a) and (b)), is subjected to proportional axisymmetric loading paths. The axis of the void is assumed to be aligned with the axis of symmetry of the matrix and the major stress is applied along the axial direction. A twodimensional axisymmetric analysis is conducted due to the axisymmetry of the problem. Exploiting additional symmetries, only one quarter of the unit-cell is analyzed (shaded region in Figs. 7(a) and (b)) subject to symmetry boundary conditions on the left and bottom boundaries, while the top and right boundaries are constrained to remain straight. The surface of the void is traction free. Special boundary conditions are imposed to ensure proportional loading by applying a constant axial strain rate while the lateral strain rate is iteratively adjusted to maintain a constant $T$. The average stress-strain response for the unit-cell is obtained by plotting the variation of the Von Mises effective stress, $\Sigma_{e}=\left|\Sigma_{33}-\Sigma_{11}\right|$, as a function of the effective strain, $E_{e}=\frac{2}{3}\left|E_{33}-E_{11}\right|$, where $\Sigma_{11}, E_{11}$ and $\Sigma_{33}, E_{33}$ are the true (Cauchy) stress and logarithmic strain of the RVE in the radial and axial directions respectively. The reader is referred to Keralavarma et al. [25] for additional details of the finite element computations. 
The effective stress-strain response of the RVE and the evolution of porosity, $f$, and void aspect ratio, $w$, computed from the evolving geometry of the void are compared against the predictions from the homogenized void growth model summarized in section 2. While the model has been shown to predict some qualitative features evidenced in cell model calculations such as the non-trivial coupling between void shape and matrix anisotropy effects [see 24], a quantitative agreement was lacking due to the limitations inherent in the choice of the composite spheroidal RVE. Most significantly, the model underestimates the evolution of porosity compared to that obtained from the unit cell calculations for moderate to high values of the stress triaxiality $(T>1)$, such as observed in the process zone ahead of a crack-tip. This is a known limitation of Gurson-like models that are derived assuming an RVE geometry that is linked to the void geometry such that the resulting criterion is independent of the RVE geometry. In a periodic array of voids as considered in the cell model calculations, homogeneous deformation rate boundary conditions apply at the boundaries of the cylindrical RVE due to the requirement of symmetry, while the deformation of spheroids confocal with the void is unlikely to be homogeneous as assumed in the derivation of the analytical model. Tvergaard [45] has shown that predictions for void growth from the Gurson model can be significantly improved by using a constant 'void growth acceleration factor' (so called Tvergaard parameter). Later finite element studies by Gologanu et al. [16] on void shape effects indicated that the value of this parameter should be a function of the void shape for non-spherical voids. In this study, we adopt the proposal of Gologanu et al. [16] and modify the axisymmetric yield criterion of equation (46) to introduce a heuristic parameter, $q_{w}$. Further, the original criterion does not account for strain hardening due to limit analysis being employed in its derivation. On the other hand, a power-law hardening model with a small hardening exponent is used in the unit cell computations for reasons of numerical stability. Therefore, strain hardening is incorporated into the analytical model using a heuristic energy balance approach for the plastic dissipation employed in previous studies [27, 38] by writing $\boldsymbol{\Sigma}: \mathbf{D}^{p}=(1-f) \bar{\sigma} d_{e q}$, where $\bar{\sigma}$ is the current yield stress of the matrix and $d_{e q}$ is the Hill equivalent strain rate defined in (38). $\bar{\sigma}$ is given by

$$
\bar{\sigma}=\sigma_{0}\left(1+\frac{\epsilon_{e q}}{\epsilon_{0}}\right)^{N}, \quad \epsilon_{e q}=\int_{0}^{t} d_{e q} \mathrm{~d} t
$$

where $\sigma_{0}$ is the initial yield stress of the matrix in a reference direction, $\epsilon_{0}$ is a reference plastic strain and $N$ is the power-law hardening exponent. Values of $\epsilon_{0}=0.002$ and $N=0.1$ are used in the present computations. Accordingly, the modified yield criterion under axisymmetric loading is written as

$$
\frac{h_{q}^{\prime} C}{\bar{\sigma}^{2}}\left(\Sigma_{33}-\Sigma_{11}+\frac{3}{2} \eta \Sigma_{h}\right)^{2}+2 q_{w}(g+1)(g+f) \cosh \left(\kappa \frac{\Sigma_{h}}{\bar{\sigma}}\right)-(g+1)^{2}-q_{w}^{2}(g+f)^{2}=0
$$

where the void growth acceleration factor $q_{w}$ in (50) is given by [see 16]

$$
q_{w}=1+2\left(q_{s}-1\right) \frac{w}{1+w^{2}}
$$


The limiting value of $q_{w}$ for a spherical cavity, $q_{s}$ is taken to be 1.6 in the following calculations.

Gologanu et al. [16] have noted that the evolution equation for the void aspect ratio (11) also requires heuristic correction to account for additional effects of porosity, void shape and stress triaxiality evidenced in finite element computations. This correction is required due to the fact that (11) is derived using the same set of trial velocity fields that realize the minimum dissipation in (12), while there is no reason to expect that these fields automatically yield the correct evolution of the void shape. We again adopt their proposal for the void shape evolution law and write

$$
\frac{\dot{w}}{w}=\phi D_{33}^{p^{\prime}}+3\left(\frac{1-3 \alpha_{1}}{f}+3 \alpha_{2}-1\right) D_{m}
$$

The parameter, $\phi$, which is taken to be a function of the porosity, void aspect ratio and the macroscopic stress triaxiality $T$ [see 16]

$$
\phi \equiv 1+\phi_{f} \phi_{e} \phi_{T}
$$

where $\phi_{f}, \phi_{e}$ and $\phi_{T}$ are functions of $f, w$ and $T$ respectively. These are given by

$$
\phi_{e}\left(e_{1}\right)=\frac{9}{2} \frac{\alpha_{1}-\alpha_{1}^{G a r}}{1-3 \alpha_{1}}, \phi_{f}(f)=(1-\sqrt{f})^{2}, \phi_{T}(T)= \begin{cases}1-\left(T^{2}+T^{4}\right) / 9 & \text { if } \Sigma_{m} \Sigma_{33}^{\prime}>0 \\ 1-\left(T^{2}+T^{4}\right) / 18 & \text { if } \Sigma_{m} \Sigma_{33}^{\prime}<0\end{cases}
$$

Expressions for $\alpha_{1}$ and $\alpha_{1}^{\text {Gar }}$ as functions of $e_{1}$ are given in Appendix A. Note that $\phi_{e}$ is a function of $w$ alone since the void eccentricity $e_{1}$ is related to $w$ via $(6)_{1}$. It is also worth noting that, in general, the heuristic parameters $q_{w}$ and $\phi$ could also depend on the material anisotropy factors for the matrix (8). However, for the purpose of the present study, this possible dependence is ignored and the proposal of Gologanu et al. [16] for spheroidal voids in an isotropic matrix is used as-is.

The model constitutive equations are integrated using a convex cutting plane integration algorithm [37, 42]. Attention is focused on the early stages of deformation, well before the onset of strain localization in the RVE. Fig. 8 shows the comparison of the stress-strain response of the unit-cell to that obtained from integration of the model constitutive equations. These results correspond to axisymmetric proportional loading with a major axial stress $\left(\Sigma_{33}>\Sigma_{11}\right)$ and a macroscopic stress triaxiality, $T=1$. The initial porosity and void aspect ratios correspond to the meshes shown in Fig. 7. The Figs. 8(a)(b) show the unit-cell and model responses respectively, for the initially prolate cavity of Fig. 7(a) and the Figs. 8(c)-(d) show the corresponding results for the initially oblate cavity of Fig. 7(b). It may be seen that the stress-strain response of the model is in close agreement with the FE results. Figs. 9(a) and 9(b) show comparison of the evolution of porosity, $f$, as a function of the effective strain in the RVE, $E_{e}$, for the initially prolate cavity of Fig. 7(a). Fig. 9(c) and 9(d) show comparison of the evolution of void aspect ratio, $w$, as a function of the effective strain for the same RVE. It is observed that the trends for the evolution of porosity and void shape with deformation for the three materials are correctly predicted by the analytical model. However, the unit-cell results for the 


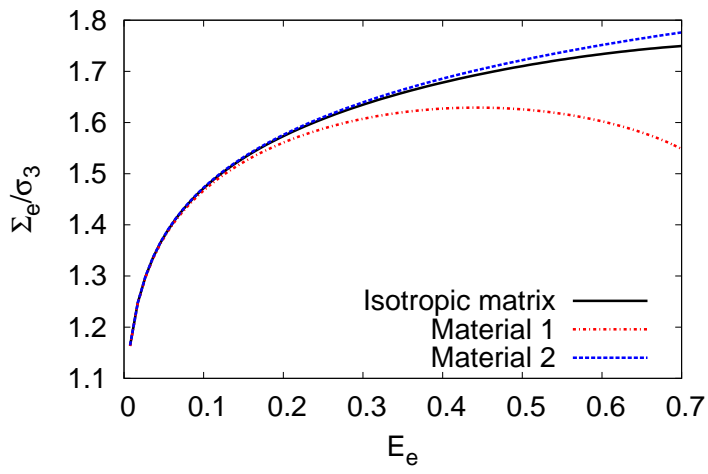

(a)

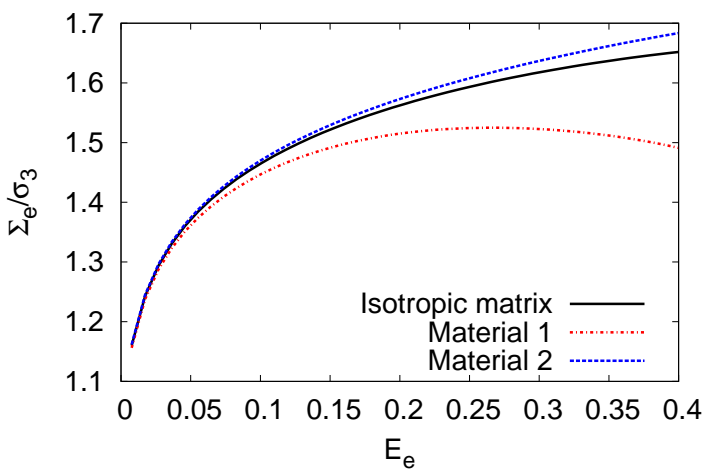

(c)

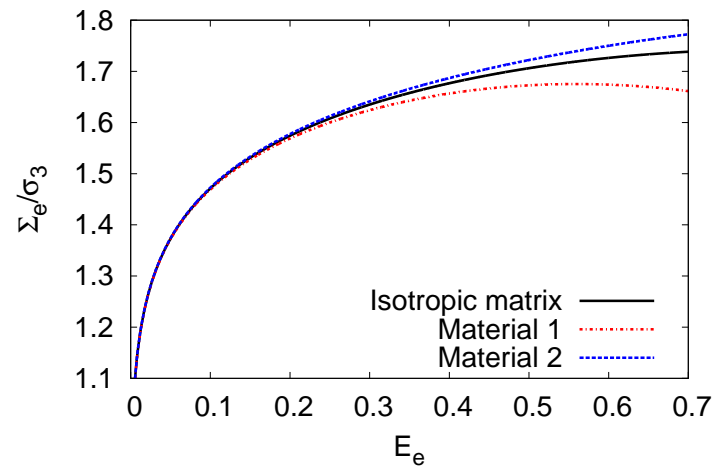

(b)

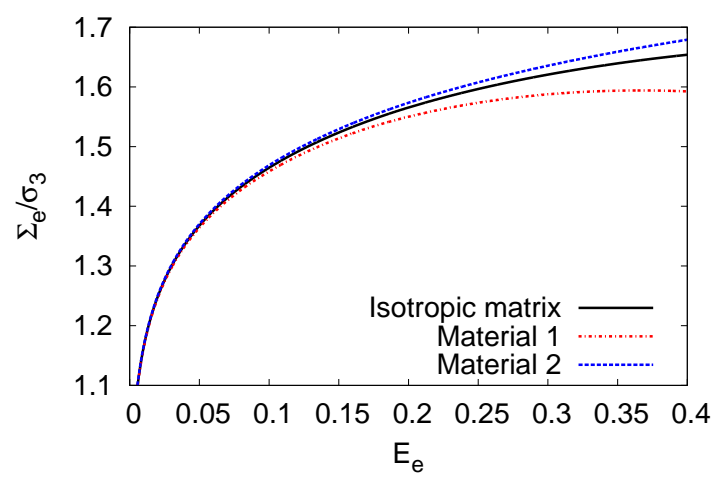

(d)

Figure 8: Comparison of the stress-strain response from the FE calculations and the analytical model for initially prolate and oblate cavities (Fig. 7) and three different materials from Table 1. The results correspond to axisymmetric proportional loading with a stress triaxiality, $T=1$. (a) FE results and (b) Model predictions for the initially prolate cavity. (c) FE results and (d) Model predictions for the initially oblate cavity. 


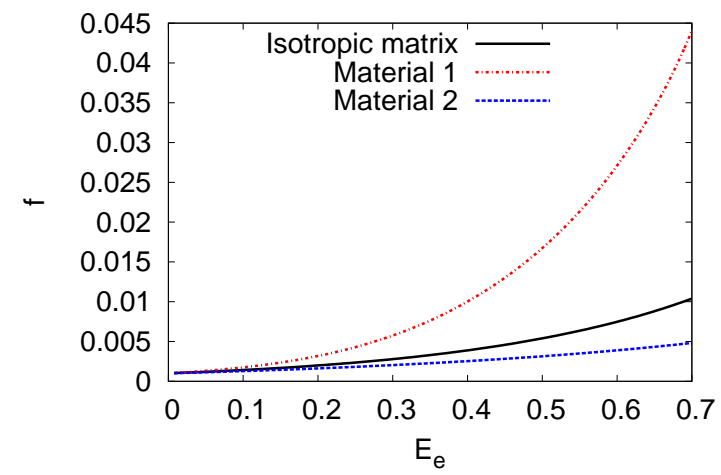

(a)

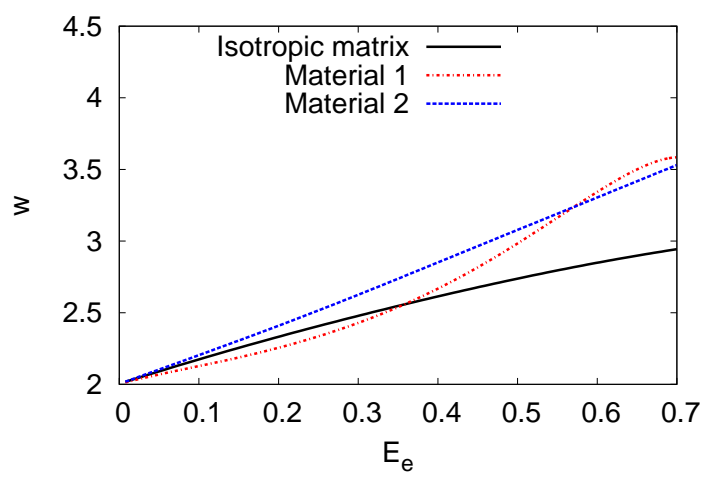

(c)

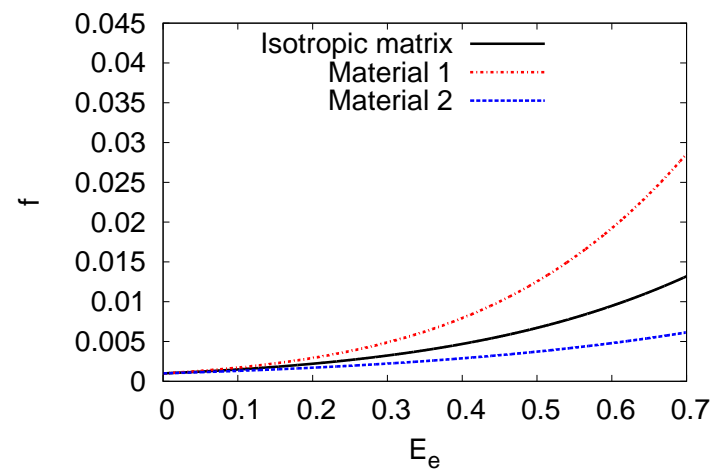

(b)

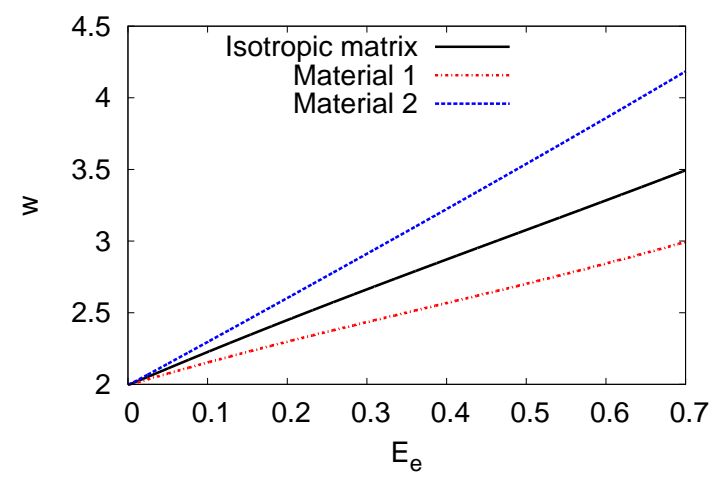

(d)

Figure 9: Comparison of the unit cell response to the model results for the evolution of $f$ and $w$, for an initially prolate cavity and three different materials from Table 1. (a) FE results for the evolution of $f$ (b) Model prediction for the evolution of $f$ (c) FE results for the evolution of $w$ (d) Model prediction for the evolution of $w$ 


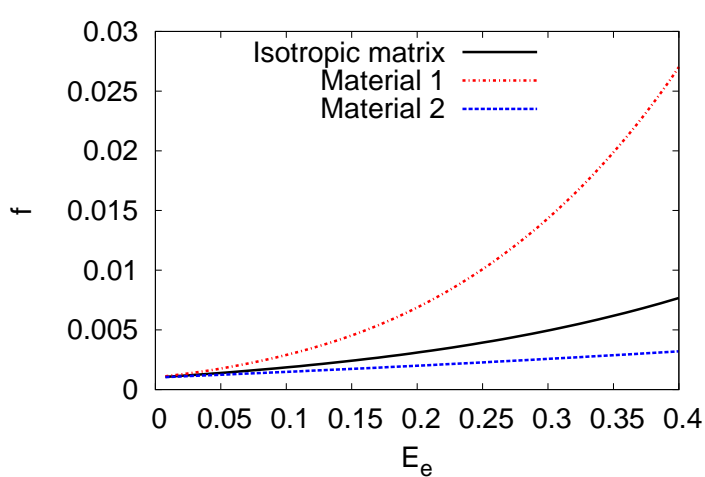

(a)

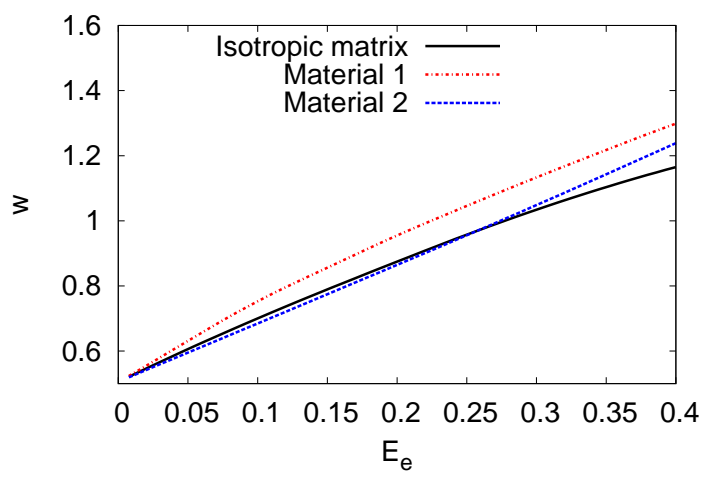

(c)

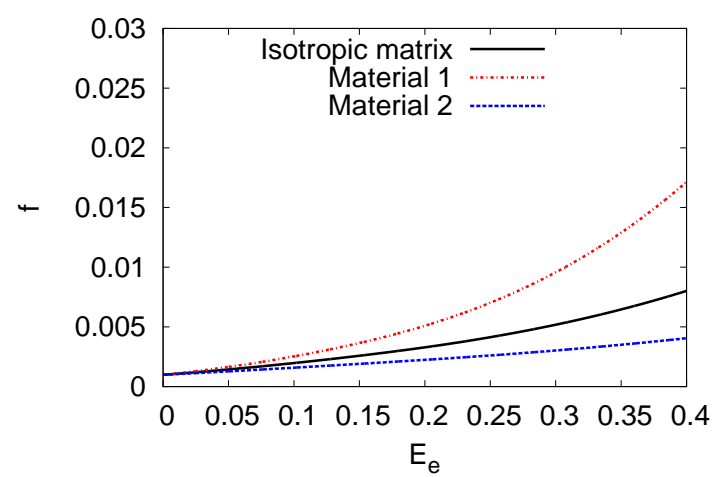

(b)

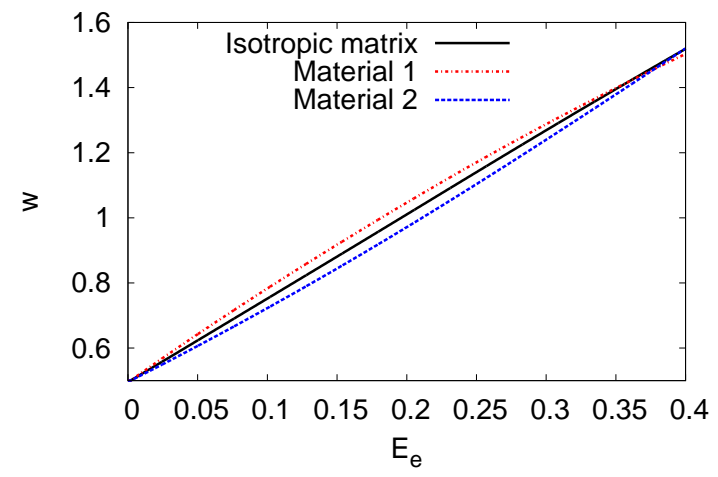

(d)

Figure 10: Comparison of the unit cell response to the model results for the evolution of $f$ and $w$, for an initially oblate cavity and three different materials from Table 1. (a) FE results for the evolution of $f$ (b) Model prediction for the evolution of $f$ (c) FE results for the evolution of $w$ (d) Model prediction for the evolution of $w$

evolution of the void aspect ratio, $w$, for Material 1 evidences an anomalous behavior at larger values of the effective strain. Examination of the corresponding deformed configuration of the unit-cell (not shown) indicates that while the void shape remains roughly spheroidal for the isotropic matrix and Material 2, this is not true for Material 1 at large strains. Since the analytical model assumes that the void remains spheroidal during the deformation, such discrepancies may be expected in the case of certain types of material anisotropy.

Figs. 10(a) and 10(b) show a similar comparison of the evolution of porosity, $f$, as a function of the effective strain in the RVE, $E_{e}$, for an initially oblate cavity. The initial porosity and void aspect ratio were $f_{0}=0.001$ and $w=1 / 2$, corresponding to Fig. 7 (b). Figs. 10(c) and 10(d) show comparison of the evolution of void aspect ratio, $w$, as a function of the effective strain for the same RVE. Again, the model correctly predicts the qualitative trends for the evolution of $f$ and $w$ with deformation for the three materials studied, together with a reasonable quantitative agreement. 


\section{Conclusion}

A numerical method to derive rigorous upper bound yield loci for spheroidal RVEs containing confocal spheroidal voids and subjected to axisymmetric stress states was developed. For a specified loading path in stress space, the yield point was obtained by conjugate gradient minimization of the plastic dissipation using a large number of axisymmetric trial velocity fields. The analytical constitutive porous metal plasticity model of Keralavarma and Benzerga [24] was also integrated to obtain the stress-strain response and evolution of the microstructural variables for specified proportional loading paths. The model predictions were compared with corresponding 'exact' results obtained from finite-element calculations. The main conclusions of this study are summarized below.

- Comparisons with numerical upper bound yield loci showed that the analytical yield criterion of equation (2) respects the upper bound character of the homogenization approach for prolate cavities as well as provides a close approximation for the numerical yield loci at all triaxialities.

- Similar comparisons for oblate cavities also yielded good agreement albeit with larger deviations from the numerical loci, possibly as a result of the different approaches used to derive the prolate and oblate criteria. Also, possible violations of the upper bound character were observed for flat voids although the differences were small in all cases considered.

- Comparison of the model predictions for the evolution of the microstructure with finite-element predictions showed good agreement for the special case of transverse isotropy. However, some deviations have been observed in cases where the void shape was found to deviate significantly from a spheroid as assumed in the analytical model.

- The analytical model thus constitutes a robust generalization of current porous metal plasticity models and can be used in large-scale simulations of ductile fracture under conditions heretofore impossible to analyze.

\section{Acknowledgments}

AAB acknowledges support from the Qatar National Research Fund (a member of Qatar Foundation) under NPRP grant No 4-1411-2-555 and from the National Science Foundation under grant CMMI-1405226. The statements made herein are solely the responsibility of the authors. 


\section{Appendix A. Expressions for the Model Parameters}

The exponential term in the criterion (2) depends on two parameters, $\kappa$ and $\alpha_{2} . \kappa$ is a measure of the pressure dependency of the yield surface, and is given by

$$
\kappa=\left\{\begin{array}{l}
\frac{3}{h}\left\{1+\frac{h_{t}}{h^{2} \ln f} \ln \frac{1-e_{2}^{2}}{1-e_{1}^{2}}\right\}^{-1 / 2} \\
\frac{3}{h}\left\{1+\frac{\left(g_{f}-g_{1}\right)+\frac{4}{5}\left(g_{f}^{5 / 2}-g_{1}^{5 / 2}\right)-\frac{3}{5}\left(g_{f}^{5}-g_{1}^{5}\right)}{\ln \frac{g_{f}}{g_{1}}}\right\}^{-1}
\end{array}\right.
$$

where

$$
g_{f} \equiv \frac{g}{g+f}, \quad g_{1} \equiv \frac{g}{g+1}
$$

and the anisotropy factors $h$ and $h_{t}$ have been defined in (8). Note that the above $\kappa$ for prolate cavities is a much simplified version of the lengthier expression given in Keralavarma and Benzerga [24]. It is verified numerically that this simplification leads to no significant loss of accuracy. The argument of the 'cosh' also depends on the term $\boldsymbol{\Sigma}: \mathbf{X}$, where $\mathbf{X}$ is defined by (4). Since the tensor $\mathbf{X}$ is coaxial with the void, this term is in fact a weighted sum of the normal stress components in the axial and transverse directions of the void, where the weights depend on the value of the parameter $\alpha_{2}$. $\alpha_{2}$ is given by

$$
\alpha_{2}=\left\{\begin{array}{c}
\frac{\left(1+e_{2}^{2}\right)}{\left(1+e_{2}^{2}\right)^{2}+2\left(1-e_{2}^{2}\right)} \\
\frac{\left(1-e_{2}^{2}\right)\left(1-2 e_{2}^{2}\right)}{\left(1-2 e_{2}^{2}\right)^{2}+2\left(1-e_{2}^{2}\right)}
\end{array}\right.
$$

The parameters $C$ and $\eta$ that appear in the square term of the yield criterion are given by

$$
\begin{gathered}
\eta=-\frac{2}{3} \frac{\kappa Q^{*}(g+1)(g+f) \operatorname{sh}}{(g+1)^{2}+(g+f)^{2}+(g+1)(g+f)\left[\kappa H^{*} \mathrm{sh}-2 \mathrm{ch}\right]}, \\
C=-\frac{\kappa h_{q}(g+1)(g+f) \operatorname{sh}}{\left(Q^{*}+\eta H^{*}\right) \eta}, \quad \operatorname{sh} \equiv \sinh \left(\kappa H^{*}\right), \quad \operatorname{ch} \equiv \cosh \left(\kappa H^{*}\right)
\end{gathered}
$$

where $H^{*} \equiv 2 \sqrt{h_{q}}\left(\alpha_{1}-\alpha_{2}\right), Q^{*} \equiv \sqrt{h_{q}}(1-f)$ and $h_{q}$ is one of the anisotropy factors defined in (8). $H^{*}$ and $Q^{*}$ depend on an additional parameter $\alpha_{1}$, given by

$$
\alpha_{1}= \begin{cases}{\left[e_{1}-\left(1-e_{1}^{2}\right) \tanh ^{-1} e_{1}\right] /\left(2 e_{1}^{3}\right)} & (\mathrm{p}) \\ {\left[-e_{1}\left(1-e_{1}^{2}\right)+\sqrt{1-e_{1}^{2}} \sin ^{-1} e_{1}\right] /\left(2 e_{1}^{3}\right)} & \text { (o) }\end{cases}
$$

Also, the heuristic parameter $\phi_{e}$ in the modified equation for void shape evolution (52) depends on $\alpha_{1}$ and another function $\alpha_{1}^{G a r}$, given by

$$
\alpha_{1}^{\text {Gar }}= \begin{cases}1 /\left(3-e_{1}^{2}\right) & (\mathrm{p}) \\ \left(1-e_{1}^{2}\right) /\left(3-2 e_{1}^{2}\right) & (\mathrm{o})\end{cases}
$$

Both $\alpha_{1}$ and $\alpha_{1}^{\text {Gar }}$ are functions of $w$ alone since $e_{1}$ is related to $w$ via $(6)_{1}$. 


\section{References}

[1] Argon, A. S., Im, J., Safoglu, R., 1975. Cavity Formation from Inclusions in Ductile Fracture. Met. Trans. A 6A, 825-837.

[2] Benzerga, A. A., 2000. Rupture Ductile des Tôles Anisotropes. Ph.D. thesis, Ecole Nationale Supérieure des Mines de Paris.

[3] Benzerga, A. A., 2002. Micromechanics of Coalescence in Ductile Fracture. J. Mech. Phys. Solids 50, 1331-1362.

[4] Benzerga, A. A., 2015. Damage Mechanics in Metal Forming. Springer, New York, Ch. Micromechanical models of ductile damage and fracture, pp. 939-962.

[5] Benzerga, A. A., Besson, J., 2001. Plastic potentials for anisotropic porous solids. Eur. J. Mech. 20 (3), 397-434.

[6] Benzerga, A. A., Besson, J., Pineau, A., 1999. Coalescence-Controlled Anisotropic Ductile Fracture. J. Eng. Mat. Tech. 121, 221-229.

[7] Benzerga, A. A., Besson, J., Pineau, A., 2004. Anisotropic ductile fracture. Part II: theory. Acta Mater. 52, 4639-4650.

[8] Benzerga, A. A., Leblond, J. B., 2010. Ductile fracture by void growth to coalescence. Adv. Appl. Mech. 44, 169-305.

[9] Benzerga, A. A., Leblond, J.-B., 2014. Effective Yield Criterion Accounting for Microvoid Coalescence. J. App. Mech. 81, 031009.

[10] Besson, J., Foerch, R., 1997. Large scale object oriented finite element code design. Comput. Methods Appl. Mech. Engrg 142, 165-187.

[11] Danas, K., Ponte Castañeda, P., 2009. A finite-strain model for anisotropic viscoplastic porous media: I-Theory. Eur. J. Mech./A Solids 28, 387-401.

[12] Gill, P., Murray, W., Wright, M., 1981. Practical Optimization. Academic Press, New York.

[13] Gologanu, M., Leblond, J.-B., Devaux, J., 1993. Approximate models for ductile metals containing non-spherical voids - case of axisymmetric prolate ellipsoidal cavities. J. Mech. Phys. Solids 41 (11), 1723-1754.

[14] Gologanu, M., Leblond, J.-B., Devaux, J., 1994. Approximate Models for Ductile Metals Containing Non-spherical Voids - Case of Axisymmetric Oblate Ellipsoidal Cavities. J. Eng. Mat. Tech. 116, 290-297.

[15] Gologanu, M., Leblond, J.-B., Perrin, G., Devaux, J., 1997. Recent Extensions of Gurson's Model for Porous Ductile Metals. In: Suquet, P. (Ed.), Continuum Micromechanics, CISM Lectures Series. Springer, New York, pp. 61-130. 
[16] Gologanu, M., Leblond, J.-B., Perrin, G., Devaux, J., 2001. Theoretical models for void coalescence in porous ductile solids - I: Coalescence in "layers". Int. J. Solids Struct. 38, 5581-5594.

[17] Gradshteyn, I., Ryzhik, I., 1980. Table of Integrals, Series, and Products. Academic Press, New York.

[18] Gurson, A. L., 1977. Continuum Theory of Ductile Rupture by Void Nucleation and Growth: Part I- Yield Criteria and Flow Rules for Porous Ductile Media. J. Eng. Mat. Tech. 99, 2-15.

[19] Han, X., Besson, J., Forest, S., Tanguy, B., Bugat, S., 2013. A yield function for single crystals containing voids. Int. J. Solids Struct. 50, 2115-2131.

[20] Hill, R., 1948. A theory of yielding and plastic flow of anisotropic solids. Proc. Roy. Soc. London A 193, 281-297.

[21] Hill, R., 1967. The essential structure of constitutive laws for metal composites and polycrystals. J. Mech. Phys. Solids 15, 79-95.

[22] Kailasam, M., Ponte Castaneda, P., 1998. A general constitutive theory for linear and nonlinear particulate media with microstructure evolution. J. Mech. Phys. Solids 46 (3), 427-465.

[23] Keralavarma, S. M., Benzerga, A. A., 2008. An approximate yield criterion for anisotropic porous media. C. R. Mecanique 336, 685-692.

[24] Keralavarma, S. M., Benzerga, A. A., 2010. A constitutive model for plastically anisotropic solids with non-spherical voids. J. Mech. Phys. Solids 58, 874-901.

[25] Keralavarma, S. M., Hoelscher, S., Benzerga, A. A., 2011. Void growth and coalescence in anisotropic plastic solids. International journal of solids and structures 48, $1696-1710$.

[26] Kondori, B., Benzerga, A. A., 2014. Effect of Stress Triaxiality on the Flow and Fracture of Mg Alloy AZ31. Metall. Mater. Trans. A 45, 3292-3307.

[27] Koplik, J., Needleman, A., 1988. Void growth and coalescence in porous plastic solids. Int. J. Solids Struct. 24 (8), 835-853.

[28] Lebensohn, R., Escobedo, J., Cerreta, E., Dennis-Koller, D., Bronkhorst, C., Bingert, J., 2013. Modeling void growth in polycrystalline materials. Acta Mater. 61, 69186932.

[29] Lee, B. J., Mear, M. E., 1992. Axisymmetric deformation of power-law solids containing a dilute concentration of aligned spheroidal voids. J. Mech. Phys. Solids 40 (8), 1805-1836. 
[30] Liu, Y. C., Johnson, L. K., 1985. Hill's Plastic Strain Ratio of Sheet Metals. Met. Trans. A 16A, 1531-1535.

[31] Madou, K., Leblond, J.-B., 2012. A Gurson-type criterion for porous ductile solids containing arbitrary ellipsoidal voids-I: Limit-analysis of some representative cell. J. Mech. Phys. Solids 60, 1020-1036.

[32] Madou, K., Leblond, J.-B., 2012. A Gurson-type criterion for porous ductile solids containing arbitrary ellipsoidal voids-II: Determination of yield criterion parameters. J. Mech. Phys. Solids 60, 1037-1058.

[33] Mandel, J., 1964. Contribution Théorique à l'étude de l'écrouissage et des Lois d'écoulement Plastique. In: $11^{\text {th }}$ International Congress on Applied Mechanics. Springer, Berlin, pp. 502-509.

[34] Monchiet, V., Cazacu, O., Charkaluk, E., Kondo, D., 2008. Macroscopic yield criteria for plastic anisotropic materials containing spheroidal voids. Int. J. Plasticity 24, $1158-1189$.

[35] Morin, L., Madou, K., Leblond, J.-B., Kondo, D., 2014. A new technique for finite element limit-analysis of hill materials, with an application to the assessment of criteria for anisotropic plastic porous solids. International Journal of Engineering Science 74, 65-79.

[36] Nielsen, K. L., Dahl, J., Tvergaard, V., 2012. Collapse and coalescence of spherical voids subject to intense shearing: studied in full 3D. Int. J. Frac. 177, 97-108.

[37] Ortiz, M., Simo, J., 1986. An Analysis of a New Class of Integration Algorithms for Elastoplastic Constitutive Relations. Int. J. Numer. Meths. Engrg. 23, 353-366.

[38] Pardoen, T., Hutchinson, J. W., 2000. An extended model for void growth and coalescence. J. Mech. Phys. Solids 48, 2467-2512.

[39] Paux, J., Morin, L., Brenner, R., Kondo, D., 2015. An approximate yield criterion for porous single crystals. European Journal of Mechanics-A/Solids 51, 1-10.

[40] Ponte Castañeda, P., Zaidman, M., 1994. Constitutive models for porous materials with evolving microstructure. J. Mech. Phys. Solids 42, 1459-1495.

[41] Rousselier, G., 1987. Ductile fracture models and their potential in local approach of fracture. Nucl. Eng. Design 105, 97-111.

[42] Simo, J., Hughes, T., 1998. Computational Inelasticity. Springer, New York.

[43] Sreeramulu, K., Biswas, P., Narasimhan, R., Mishra, R. K., 2013. Ductile fracture by multiple void growth and interaction ahead of a notch tip in polycrystalline plastic solids. Int. J. Frac., 1-17. 
[44] Suquet, P., 1982. Plasticité et homogénéisation. Thése de Doctorat d'Etat, Université Pierre et Marie Curie - Paris VI.

[45] Tvergaard, V., 1981. Influence of voids on shear band instabilities under plane strain conditions. Int. J. Frac. 17, 389-407.

[46] Tvergaard, V., 2014. Effect of initial void shape on ductile failure in a shear field. Mech. Mater., Submitted.

[47] Tvergaard, V., Needleman, A., 1984. Analysis of the cup-cone fracture in a round tensile bar. Acta Metall. 32, 157-169.

[48] Yerra, S. K., Tekoglu, C., Scheyvaerts, F., Delannay, L., Van Houtte, P., Pardoen, T., 2010. Void growth and coalescence in single crystals. Int. J. Solids Struct. 47, 1016-1029. 